\title{
Chemistry of Cysteine Assembly on Au(100): Electrochemistry, in situ STM and Molecular Modeling
}

Engelbrekt, Christian; Nazmutdinov, Renat R.; Zinkicheva, Tamara; Glukhov, Dmitrii; Yan, Jiawei; Mao, Bingwei; Ulstrup, Jens; Zhang, Jingdong

\section{Published in:}

Nanoscale

Link to article, DOI:

10.1039/C9NR02477H

Publication date:

2019

Document Version

Peer reviewed version

Link back to DTU Orbit

Citation $(A P A)$ :

Engelbrekt, C., Nazmutdinov, R. R., Zinkicheva, T., Glukhov, D., Yan, J., Mao, B., Ulstrup, J., \& Zhang, J. (2019). Chemistry of Cysteine Assembly on $\mathrm{Au}(100)$ : Electrochemistry, in situ STM and Molecular Modeling. Nanoscale, 11(37), 17235-17251. https://doi.org/10.1039/C9NR02477H

\section{General rights}

Copyright and moral rights for the publications made accessible in the public portal are retained by the authors and/or other copyright owners and it is a condition of accessing publications that users recognise and abide by the legal requirements associated with these rights.

- Users may download and print one copy of any publication from the public portal for the purpose of private study or research.

- You may not further distribute the material or use it for any profit-making activity or commercial gain

- You may freely distribute the URL identifying the publication in the public portal 


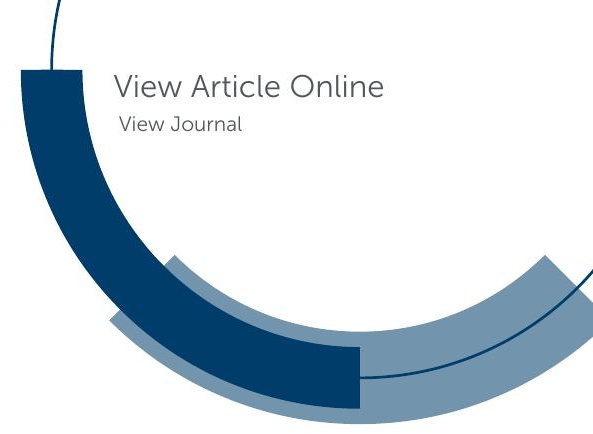

\section{Accepted Manuscript}

This article can be cited before page numbers have been issued, to do this please use: C. Engelbrekt, R. Nazmutdinov, T. T. Zinkicheva, D. V. Glukhov, J. Yan, B. Mao, J. Ulstrup and J. Zhang, Nanoscale, 2019,

\section{Nanoscale}

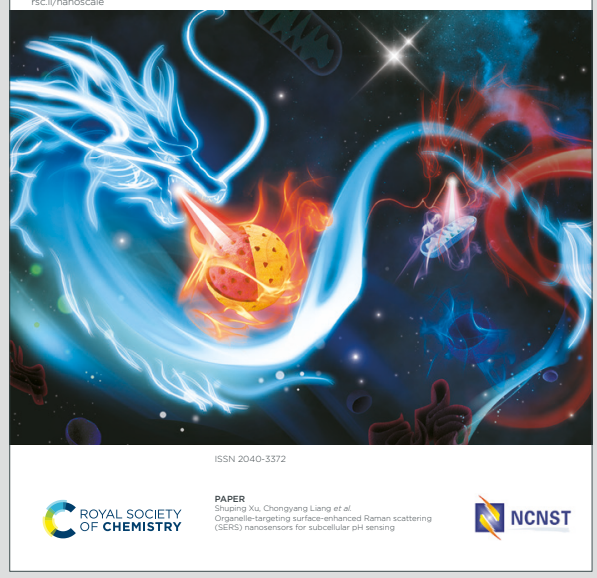

This is an Accepted Manuscript, which has been through the Royal Society of Chemistry peer review process and has been accepted for publication.

Accepted Manuscripts are published online shortly after acceptance, before technical editing, formatting and proof reading. Using this free service, authors can make their results available to the community, in citable form, before we publish the edited article. We will replace this Accepted Manuscript with the edited and formatted Advance Article as soon as it is available.

You can find more information about Accepted Manuscripts in the Information for Authors.

Please note that technical editing may introduce minor changes to the text and/or graphics, which may alter content. The journal's standard Terms \& Conditions and the Ethical guidelines still apply. In no event shall the Royal Society of Chemistry be held responsible for any errors or omissions in this Accepted Manuscript or any consequences arising from the use of any information it contains. 


\section{Chemistry of Cysteine Assembly on Au(100): Electrochemistry, in situ STM and Molecular Modeling}

Christian Engelbrekt ${ }^{1}$, Renat R. Nazmutdinov ${ }^{2}$, Tamara Zinkicheva, ${ }^{2}$ Dmitrii Glukhov ${ }^{2}$, Jiawei Yan $^{3}$, Bingwei $\mathrm{Mao}^{3}$, Jens Ulstrup ${ }^{1,2}$, Jingdong Zhang ${ }^{1 *}$

1. Department of Chemistry, Building 207, Technical University of Denmark, 2800 Kgs. Lyngby, Denmark.

2. Kazan National Research Technological University, K. Marx Str. 68, 420015 Kazan, Republic of Tatarstan, Russian Federation.

3. State Key Laboratory of Physical Chemistry of Solid Surfaces and College of Chemistry and Chemical Engineering, Xiamen University. Xiamen 361005, Fujian, China.

Cysteine monolayers on Au(100), adsorption dynamics, network assembling, electrochemical interface, DFT

Cysteine (Cys) is an essential amino acid with a carboxylic acid, an amine and a thiol group. We have studied the surface structure and adsorption dynamics of L-cysteine adlayers on $\mathrm{Au}(100)$ from aqueous solution using electrochemistry, high-resolution electrochemical scanning tunnelling microscopy (in situ STM), and molecular modelling. Cys adsorption on this low-index Au-surface has been much less studied than Cys adsorption on $\mathrm{Au}(111)$ - and $\mathrm{Au}(110)$-electrode surfaces. Chronopotentiometry was employed to monitor the adsorption dynamics at sub-second resolution and showed that adsorption is completed in 30 minutes at Cys concentrations above $100 \mu \mathrm{M}$. Two consecutive steps could be fitted to these data. Two separate reductive desorption peaks of Cys adlayers on $\mathrm{Au}(100)$ with a total coverage of $2.52( \pm 0.15) \times 10^{-10} \mathrm{~mol} / \mathrm{cm}^{2}$ were observed. In situ STM showed that adsorbed Cys is organized in stripes with "fork-like" features which co-exist in $(11 \times 2)-2$ Cys and $(7 \times 2)-2$ Cys lattices, quite differently from Cys adsorption on $\mathrm{Au}(111)$-electrode surfaces. Stripe structures with bright STM contrast in the center suggest that a second Cys adlayer on top of a first adlayer is formed, supporting the dual-peak reductive desorption of Cys adlayers. In addition, monolayers of both pure L-Cys, pure D-Cys and a 1:1 racemic mixture of $\mathrm{L}$ - and $\mathrm{D}$-Cys on $\mathrm{Au}(100)$ were studied. Virtually identical macroscopic electrochemical features were found, but in situ STM discloses many more defects for the racemic mixture than for the pure enantiomers due to structural mismatch of L- and D-Cys. Density functional theory (DFT) calculations combined with a cluster model for the $\mathrm{Au}(100)$ surface were carried out to investigate the adsorption energy and geometry of adsorbed monomer and dimer Cys species in different orientations, with detailed attention to the chirality effects. Optimized DFT geometries were used to construct model STM images, and kinetic Monte Carlo simulations undertaken to illuminate the growth of adsorbate rows and the mechanism of the adlayer formation as well as the Cys adsorption patterns specific to the $\mathrm{Au}(100)$-electrode surface.

\section{Introduction}

Among the 20 essential amino acids, Lcysteine (L-Cys) $\left(\mathrm{HO}_{2} \mathrm{CCH}\left(\mathrm{NH}_{2}\right) \mathrm{CH}_{2} \mathrm{SH}\right)$ is the only one with a free thiol (-SH) group in addition to the carboxylate and ammonium groups. L-Cys is crucial in protein chemistry 
and other biological function, ${ }^{1,2}$ and widely used in food production, cosmetics, pharmaceutical industries, ${ }^{3}$ and as a linker molecule for immobilization of metal nanoparticles on gold and graphene electrode surfaces. ${ }^{4,5}$

The reactivity of L-Cys depends on the local environment. ${ }^{1}$ Electrochemistry has long been used in order to understand the formation of cystine with a disulfur bridge (S-S-), and oxidation of the thiol group to higher sulfur oxidation states such as $\mathrm{RSO}_{3}{ }^{-}$ . 6-12 L-Cys on metal electrodes gives a number of voltammetric peaks, ${ }^{9,11}$ but is less active on carbon electrodes and here requires mediator molecules or metallic nanoparticles as electrocatalysts for oxidation or reduction, 7,10 mainly due to strong adsorption of L-Cys and formation of metal-S chemical bonds. Cys can assemble spontaneously on gold surfaces either in vacuum or in aqueous solution. Depending on solution $\mathrm{pH}$, self-assembled monolayers (SAMs) of Cys provide both carboxylate and amine binding groups on a solid surface, onto which biological molecules such as proteins can be immobilized in well controlled orientation and interfacial electron transfer function retained. ${ }^{13,14}$ Structures and properties of Cys SAMs have been investigated using methods that include scanning tunnelling microscopy (STM), X-ray photoelectron spectroscopy (XPS), the quartz crystal microbalance, electrochemistry, and infrared spectroscopy both in aqueous solution and ultra-high vacuum (UHV). ${ }^{15-29}$

Well-defined Cys SAM structures are only achieved on atomically flat substrates, single-crystal gold surfaces in particular. ${ }^{17,18,20-25}$ Low-index crystal facets such as $A u(111)$ and $A u(110)$ have been employed, and molecular or even submolecular structural information achieved. STM both in vacuum and liquid environment has reached a level of atomic resolution ideally suitable for visualizing molecular packing. ${ }^{30}$ Notably the organization of Cys molecules in SAMs is sensitive to the environments. In aqueous solutions, Cys is adsorbed chemically on $\mathrm{Au}(111)$ forming highly ordered monolayers with $(\sqrt{3} \times$ $\sqrt{ } 3) \mathrm{R} 30^{\circ},(3 \sqrt{ } 3 \times 6) \mathrm{R} 30^{\circ}$, and $(4 \times \sqrt{ } 7) \mathrm{R} 19^{\circ}$ cluster-like networks depending on both supporting electrolyte and $\mathrm{pH}^{16,18,19,31}$ Cys SAM formation is accompanied by lift of reconstruction in the metallic surface structure even though the surface structures underneath the adlayers are different. Physisorption of Cys is found in UHV at room temperature and chemisorption at higher temperature. ${ }^{21-23} \mathrm{~A}$ variety of 
molecular surface organization forms can, moreover co-exist on Au(111) in UHV. These can be disordered Cys islands or extended domains of ordered quadratic and trimer Cys adlayers. ${ }^{23}$ For example, Cys forms pairs of molecules or clusters of eight molecules in UHV on $\mathrm{Au}(110),{ }^{21-23}$ while a highly ordered $\mathrm{c}(2 \times 2)$ dense monolayer with two Cys molecules in each unit cell is found in aqueous buffer. ${ }^{25}$ Sub-molecular resolution was achieved by the visualization of three sub-molecular spots corresponding to the three functional groups of each Cys molecule by in situ STM. ${ }^{25}$

It has been a puzzle that only the L-forms of amino acids are found in nature, even though the chemical nature of $L$ - and $D$ enantiomers is identical. Chiral effects has been discovered only in pair formation of Cys molecules on the $\mathrm{Au}(110)$ surface in UHV. ${ }^{20}$ Cys dimers on $\mathrm{Au}(111)$ in aqueous environment were not found, ${ }^{25}$ but Cys dimer formation perpendicular to polycrystalline gold surfaces was suggested based on surface plasmon resonance (SPR) spectroscopy. ${ }^{32}$

Molecular level understanding of Cys SAM formation on metal surfaces is a challenge. Attempts based on DFT calculations were reported by Kühnle and associates. ${ }^{33-36} \mathrm{~A}$ stable double row of cyclic Cys molecular dimers on $A u(110)$ in UHV was found, supported by UHV STM data. ${ }^{33}$ Cys molecules were also found to diffuse freely along the $A u(111)$ surface by combining experimental techniques (XPS, ultraviolet photoelectron spectroscopy, low energy electron diffraction, and STM) with density functional theory (DFT), resembling a 2Dmolecular gas. ${ }^{26}$ No stable cyclic dimers were observed, and formation of single molecular rows was concluded to be more likely. Different types of Cys-Cys dimers (radical forms) on $\mathrm{Au}(110)$ were also investigated $^{35}$. Some dimers could be considered as nucleation centers for the SAM formation. Adsorption of dimers and clusters of Cys molecules at the $\mathrm{Au}(110)$ surface studied by DFT have also been reported. ${ }^{37,38}$ Chiral effects on the adsorption of cyclic Cys dimers (L-L, D-D and L-D) were furthermore investigated by Paci, Cordeiro, and associates, ${ }^{34,36}$ but the solvent environment was not addressed.33-36 Solvation is, however, crucial as even the mere identity of the Cys molecule is quite different in UHV (neutral molecule) and in polar solvents (zwitter ion). The energy barrier of the $\mathrm{S}-\mathrm{H}$ bond cleavage in dissociative adsorption of a Cys molecule on the $A u(111)$ surface was furthermore calculated, ${ }^{34,39}$ based on the method of 
reactive molecular dynamics with the solvent incorporated explicitly.

In this report, we address the formation of a Cys adlayer on $\mathrm{Au}(100)$ in phosphate buffer, aiming at both dynamic and structural molecular scale mapping, using a combined and comprehensive experimental and theoretical approach. $\mathrm{Au}(100)$ was chosen, as Cys adsorption on this surface has been much less studied than on the other lowindex Au-surfaces, ${ }^{40-49}$ but offers clues to new and unique Cys packing modes due to the square symmetry of the $\mathrm{Au}(100)$ crystalline surface structure. Reconstruction with "hex" transition on clean $\mathrm{Au}(100)$ is commonly found after annealing or under electrochemical potential control. ${ }^{41}$ Chemical adsorption and interaction between Cys molecules and surface gold atoms lifts the $\mathrm{Au}(100)$ reconstruction, reorganizing the surface Au-atoms into a square pattern.

The Cys coverage on $\mathrm{Au}(100)$ was recorded voltammetrically by reductive desorption which is based on cleavage of the S-Au bonds at negative potential in alkaline solution. The macroscopic time dependent adsorption was investigated by chronopotentiometry and framed microscopically, first by high-resolution in situ STM mapping the Cys adlayers on $\mathrm{Au}(100)$ in phosphate buffer solution. Chiral effects of Cys adlayers of pure L- and D-Cys as well as a racemic mixture were all included. Secondly, both DFT calculations combined with a cluster model and Monte Carlo simulations were used to elucidate microscopic details of the adsorption process. The work offers a comprehensive view of Cys assembly and packing on $\mathrm{Au}(100)$ at the molecular and sub-molecular levels of resolution.

\section{Experimental methods and theoretical calculation}

2.1 Chemicals. D-Cys (99\%) was from Aldrich and L-Cys (99.5\%) from Fluka. $\mathrm{KH}_{2} \mathrm{PO}_{4}$ (99.99 \%, CAS 7778-77-0, Aldrich) was supporting electrolyte. Freshly prepared $0.10 \mathrm{M} \mathrm{NaOH}$ (99.99\%, Sigma-Aldrich) was used for reductive desorption.

\subsection{Electrochemical methods}

Cyclic voltammetry (CV) and chronopotentiometry (zero current) were carried out using a PGSTAT12 potentiostat (Metrohm Autolab B.V., Netherlands). An in-house 3electrode glass cell with working (WE), counter (CE), and reference electrode (RE) was used for CV. Chronopotentiometry was employed to record the potential difference between the WE and RE over time during Cys adsorption. The WEs included singlecrystal gold and graphite electrodes. $\mathrm{Au}(100), \quad \mathrm{Au}(111)$ and $\mathrm{Au}(110)$ bead 
electrodes were prepared by Hamelin's method, 50 and checked by $\mathrm{CV}$ in $0.10 \mathrm{M}$ $\mathrm{H}_{2} \mathrm{SO}_{4}{ }^{18,50}$. Annealing at $860{ }^{\circ} \mathrm{C}$ and electrochemical polishing were used for cleaning the $\mathrm{Au}(100), \mathrm{Au}(111)$ and $\mathrm{Au}(110)$ surfaces. Prior to electrochemical and in situ STM measurements, the gold electrodes were annealed in a hydrogen flame and quenched in Millipore water saturated with $\mathrm{H}_{2}$. Basal plane (BPG, $\varnothing 5 \mathrm{~mm}$ ) and edge plane graphite (EPG, $\varnothing 5 \mathrm{~mm}$ ) electrodes from Pine Instrument Company (Grove, USA) were used as reference WEs in chronopotentiometry. BPG and EPG electrodes were freshly cleaned by mechanical polishing on SiC-papers followed by polishing with $1.0,0.3$ and $0.1 \mu \mathrm{m} \mathrm{Al}_{2} \mathrm{O}_{3}$ slurry on a felt disk before ultrasonication twice in Millipore water. A freshly prepared reversible hydrogen electrode (RHE) calibrated vs. a saturated calomel electrode (SCE) was used as RE for all electrochemical measurements. All potentials are referenced to SCE. The inhouse setup for chronopotentiometric monitoring of cysteine assembly in $25 \mathrm{mM}$ $\mathrm{KH}_{2} \mathrm{PO}_{4}$ is described in a previous report ${ }^{51}$. CV was used to monitor both adsorption and reductive desorption of Cys adlayers in 25 $\mathrm{mM} \mathrm{KH}_{2} \mathrm{PO}_{4}$ and $0.1 \mathrm{M} \mathrm{KOH}$, respectively.

\subsection{In situ STM}

A PicoSPM STM (Agilent) instrument with an in-house Teflon cell was employed for in situ STM. Platinum wires served as both CE and RE. The RE was calibrated vs a SCE after each experiment. Electrochemically etched W tips $(\varnothing 0.25 \mathrm{~mm}$ ) coated with Apiezon wax were utilized as STM probes. Au(100) disc electrodes (SPL, The Netherlands) were used for in situ STM. STM samples were prepared in the same way as for electrochemistry, i.e. the cleaned $\mathrm{Au}(100)$ electrodes were annealed in a hydrogen flame and quenched in Millipore water saturated with $\mathrm{H}_{2}$, then mounted in the STM cell with freshly prepared $25 \mathrm{mM} \mathrm{KH}_{2} \mathrm{PO}_{4}$. Freshly prepared L-, D-, or L,D-cysteine solution was injected into the cell under potential control. All STM images were recorded under electrochemical control.

Glassware, and electrochemical and STM cells were cleaned by boiling in $15 \% \mathrm{HNO}_{3}$ and washed copiously in Millipore water. All supporting electrolyte and Cys solutions were freshly prepared prior to each experiment.

\subsection{Computational Methods}

2.4.1 Molecular structures and Au-surface molecular models

In comparison to $\mathrm{Au}(110)$ or $\mathrm{Au}(111),{ }^{25}$ the in situ STM data show completely different Cys lattice at the $\mathrm{Au}(100) /$ electrolyte interface. Clear stripes (rows) of bright STM contrasts separated by dark areas are observed, sometimes with a 
"fork-like" structure. Using periodical DFT is therefore a complex, combinatorial issue.

The nature of the interaction between gold and Cys monomer forms has been theoretically addressed in detail. $24,37,38,52,53$ Our main emphasis was, however on the adsorption of different Cys dimers considered as 2D-"nucleation" centers which trigger further growth of the rows. The $\mathrm{Au}(100)$ metal surface was modelled by twolayer clusters of different size $\left(\mathrm{Au}_{25}(16+9)\right.$, $\left.\mathrm{Au}_{30}(15+15), \mathrm{Au}_{32}(20+12)\right)$. Primary attention was given to a comparative study that acknowledges the well-known shortcomings of the cluster model..$^{54} \mathrm{~A}$ merit is, however, that solvent effects can be incorporated both explicitly and implicitly (in terms of a selfconsistent reaction field). The quantum chemical calculations were performed at the DFT level with the hybrid exchange-correlation functional b3pw91 as implemented in the Gaussian 09 program suite. ${ }^{55}$ The valence orbitals of the $\mathrm{Au}$ atom were described by a basis set of double-zeta (DZ) quality, while the inner electrons were included in the Hay and Wadt effective core potential (LanL2). ${ }^{56}$ The standard basis set 631(d) was used to describe the electrons in the $\mathrm{S}$, $\mathrm{N}, \mathrm{O}, \mathrm{C}$ and $\mathrm{H}$ atoms. The open shell systems were treated in unrestricted formalism. The geometry of adsorbed species was fully optimized without symmetry restrictions. The natural population analysis (NPA) was employed to calculate the atomic charges. Water molecules were not included directly in the first solvation sphere, but the aqueous medium was addressed by the PCM (Polarized Continuum Model) as implemented in the Gaussian 09 package..$^{55} \mathrm{~A}$ value of 78.4 was taken for the static dielectric constant and a value of 1.78 for the fast solvent modes. Non-electrostatic (cavitation, dispersion and repulsion) contributions to the solvation free energy were calculated as well. The program ChemCraft ${ }^{57}$ was used for the visualization of the optimized adsorbate geometry.

\subsubsection{In situ STM contrasts}

The STM contrasts (constant current mode) were calculated in the framework of the quantum chemical model previously developed. ${ }^{24,25}$ The electron transfer (tunnelling) process was treated
View Article Online
DOI: 10.1039/C9NRO2477H as a bridge-assisted superexchange process with the electrode/Cys transition adiabatic, and the Cys/STM tip transition diabatic. The STM tip was represented by a tungsten atom, and the optimized geometry of the adsorbed Cys species used. The influence of the metal surface was not addressed directly. In the weak-coupling limit of tip-molecule interaction, the current is proportional to the squared electronic coupling factor $V_{i f}$ (resonance integral) calculated by twolevel perturbation theory. ${ }^{24,25}$

\subsubsection{Monte Carlo (MC) simulations}

A squared lattice including $100 \times 100$ surface sites was used in the MC simulations. The total number of steps amounts to 8000000 . At first all sites were assumed to be occupied by neutral Cys molecules. In subsequent iterations the weakly adsorbed Cys molecules were transformed to Cys radical and Cys-zwitter ionic forms with specified probabilities $\left(P_{m r}\right.$ and $P_{r z}$, see Figure S1). The computation scheme resembles that usually employed in kinetic MC modeling. A random number $\xi_{1}$ between 0 and 1 is first generated and compared with the pre-specified $P_{m r}$ value. If $\xi_{1}>P_{m r}$, the attempt is considered successful. A second random value $\xi_{2}$ is then generated in a second step and compared with the $P_{r z}$ value; if $\xi_{2}>\mathrm{P}_{\mathrm{rz}}$ such an attempt is kept as successful etc. The formation of the zwitterion from the adsorbed Cys radical is modelled similarly. The four neighbors of each site (right, left, up and down) or three (two) neighbors (for the sites on the 
boundary) are analyzed in subsequent computational loops. The input probabilities, $P_{r m}$ and $P_{r r}$ (Figure S1) control the formation of rad - mol and rad - rad Cys dimers in the presence of appropriate neighbors in the lattice knots. In turn, the probabilities $\mathrm{P}_{z z}$ and $\mathrm{P}_{\mathrm{zv}}$ describe the appearance of other Cys dimers, zwitter rad - zwitter rad (both species are adsorbed in the first monolayer), and zwitter rad (the first layer) - zwitter mol (the second layer) pairs, respectively. These forms are denoted as planar and vertical Cys zwitter ionic dimers (ZZp and Zv). Single dimers of both types were treated as nucleation centers which initiate the growth of rows consisting of Cys zwitter radicals or vertical dimers $\mathrm{Zv}$, as well as assigning the row direction. Such rows were assumed to grow only in vertical or horizontal directions of the $2 \mathrm{D}$ lattice that fits the symmetry of the (100) metal face. The growing rows accumulate all single Cys zwitter rad forms. Additionally, rows that encounter Cys radrad and rad-mol dimers as neighbors, break these forms with unit probability and amalgamate with the Cys radicals to single zwitter ions. The parallel rows then form arrays at the surface.

We excluded crossing rows, i.e. when a growing row meets another one, the growth stops. We assumed, however that if a row of
Cys vertical dimers meets a ZZp row (array), growth continues with a certain probability $\mathrm{P}^{*} z \mathrm{v}$. To describe a similar event (i.e. when an array of Cys zwitter ions in planar orientation crosses a strip formed by the Cys vertical dimers and continues its growth), we introduced another probability, $\mathrm{P}^{*} \mathrm{zZ}$. Another condition to stop the growth is meeting with a Cys molecule. An original code written in the program suite Matlab was used in all simulations. We varied all input probabilities within certain intervals; in the final simulations probability values were used which yield the most distinct and longest rows, Figure S1. Test simulations were performed also for larger lattices, $(150 \times 150$ and $250 \times 250$ sites) and found to give results very similar to those for the $100 \times 100$ ensemble. We note that the different Cys chiral forms ( $D$ and $L$ ) cannot be unambiguously distinguished in our computational scheme. The influence of the Cys concentration in the solution bulk on the SAM formation was not addressed either.

\section{Results and Discussion}

\subsection{Electrochemistry}

Electrochemical techniques are core techniques in exploring solid-liquid interfaces. Voltammetry and chronopotentiometry were used in this work to study Au-S Cys chemisorption, Cys coverage, as well as Cys adsorption 
dynamics. Cyclic voltammograms of bare $\mathrm{Au}(100)$ give two sharp and small anodic peaks in $25 \mathrm{mM} \mathrm{KH}_{2} \mathrm{PO}_{4}$ (pH 4.7). The peak at $0.23 \mathrm{~V}$ is caused by anion adsorption, whereas the anodic peak at $0.07 \mathrm{~V}$ and a broad cathodic peak at $0.04 \mathrm{~V}$ are due to lift and formation of surface reconstructions respectively, Figure 1. Similar voltammetric patterns were found for $\mathrm{Au}(111)$ and $\mathrm{Au}(110)$ as well, $24,25,58$ characteristic of adsorption of anions and formation of a reconstructed low index gold surface. ${ }^{59}$ Cys adlayers give a featureless double layer signal between 0.40 and $+0.45 \mathrm{~V}$, (red curve, Figure 1) suggesting stronger adsorption than the electrolyte anions and lift of surface reconstruction, i.e. chemisorption of Cys on $\mathrm{Au}(100)$. Peaks caused by oxidation and reduction of Cys adlayers on $\mathrm{Au}(100)$ occur at potentials more positive than $0.50 \mathrm{~V}$ and more negative than $-0.4 \mathrm{~V}$, respectively.

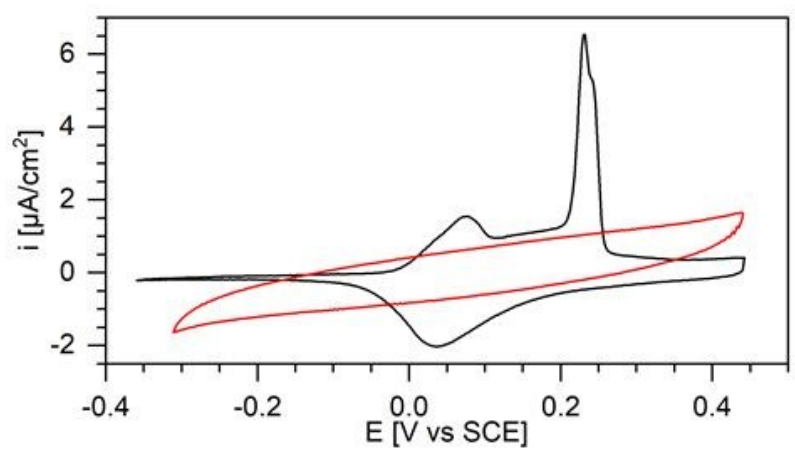

Figure 1. Cyclic voltammograms of bare $\mathrm{Au}(100)$ (black) and LCys SAM on $\mathrm{Au}(100)$ (red) in $25 \mathrm{mM} \mathrm{KH}_{2} \mathrm{PO}_{4}$ (pH 4.66). Scan rate: $50 \mathrm{mV} / \mathrm{s}$. $\mathrm{Au}(100)$ soaked in $1.6 \mathrm{mM} \mathrm{L}$-Cys for 5.75 hours.

Reductive desorption was carried out using $0.10 \mathrm{M} \mathrm{NaOH}$ to avoid interference from dihydrogen evolution, Figure 2. Cleavage of
Au-S bonds occurs at negative potentials in basic solution, giving a strong cathodic signal. The charge under the cathodic peak, notably composed of two peaks, at $-0.67 \mathrm{~V}$ and $-0.96 \mathrm{~V}$ gives Au-S coverage with charges of $(7.0-8.8) \times 10^{-6}$ and $(1.65-1.82) \times 10^{-}$ ${ }^{5} \mathrm{C} \mathrm{cm}^{-2}$, respectively, Figure 2 . This is very different from the single cathodic Cys peak at $-0.705 \mathrm{~V}$ on $\mathrm{Au}(111)^{18}(3.7 \pm 0.4) \times 10^{-5} \mathrm{C}$ $\mathrm{cm}^{-2}$ (coverage $(4.0 \pm 0.4) \times 10^{-10} \mathrm{~mol} \mathrm{~cm}^{-2}$ ) and the $-0.71 \mathrm{~V}(6.74 \pm 0.3) \times 10^{-10} \mathrm{~mol} \mathrm{~cm}^{-2}$ Cys peak on $\mathrm{Au}(110) .{ }^{24}$ Two cathodic peaks, a sharp peak at $-0.70 \mathrm{~V}(\mathrm{vs} \mathrm{Ag} / \mathrm{AgCl})$, and a large but broad peak at $-1.05 \mathrm{~V}$ were reported for Cys reductive desorption on polycrystalline gold, though the origin of the $1.05 \mathrm{~V}$ peak was not clear. ${ }^{19}$ In comparison, reductive desorption of $n$-butanethiol on $\mathrm{Au}(111)$ and $\mathrm{Au}(100)^{60}$ gives peaks at -0.99 $\mathrm{V}\left(78.6 \times 10^{-6} \mathrm{C} \mathrm{cm}^{-2}\right)$ and $-1.03 \mathrm{~V}\left(106 \times 10^{-6}\right.$ $\mathrm{C} \mathrm{cm}^{-2}$ ), very similar to the Cys reductive peak on $\mathrm{Au}(111)$ and $\mathrm{Au}(100) \cdot{ }^{61,62} \mathrm{~A} 260 \mathrm{mV}$ difference was reported for reductive desorption of 4-pyridinethiol on $\mathrm{Au}(111)$ at $0.50 \mathrm{~V}$ (coverage $5.0 \times 10^{-10} \mathrm{~mol} \mathrm{~cm}^{2}$ ) and $\mathrm{Au}(100)$ at $-0.76 \mathrm{~V}$ (coverage $5.7 \times 10^{-10} \mathrm{~mol}$ $\left.\mathrm{cm}^{-2}\right) .{ }^{62,63}$ All this is different from reductive desorption of Cys on $\mathrm{Au}(100)$. The total charge of the two cathodic peaks is $(2.5 \pm 0.3) \times 10^{-5} \mathrm{C} \mathrm{cm}^{-2}$, which is much smaller than for the network cluster-structure of Cys on $\mathrm{Au}(111),{ }^{18}$ suggesting stronger 
intermolecular interaction on the latter surface. The $290 \mathrm{mV}$ difference between the two cathodic peaks indicates that Cys on $\mathrm{Au}(100)$ displays at least two different adsorption modes with significantly different Au-S binding energies. The cathodic peak at $-0.67 \mathrm{~V}$ is also not a single sharp peak, but a convolution of two peaks, both close to -0.71 $V$ for Cys on $\mathrm{Au}(111)^{18}$ and $\mathrm{Au}(110)^{24}$. Adsorption reflected by this peak is therefore similar to Cys adsorption on $\mathrm{Au}(111)$ and $\mathrm{Au}(110)$, i.e. a monolayer of chemisorbed Cys.
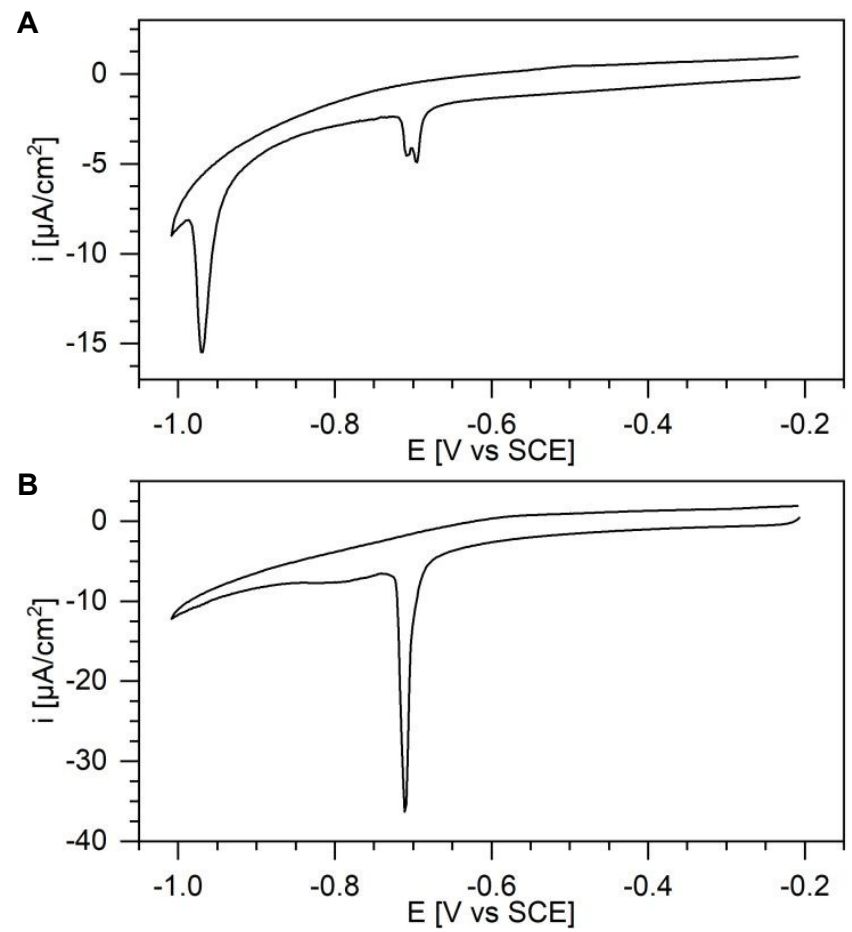

Figure 2. Reductive desorption of L-Cys SAMs on (A) $\mathrm{Au}(100)$ and (B) $\mathrm{Au}(111)$ in $0.10 \mathrm{M} \mathrm{NaOH}$. Scan rate $10 \mathrm{mVs}^{-1}$. $\mathrm{Au}(100)$ and $\mathrm{Au}(111)$ soaked in $1.03 \mathrm{mM}$ L-Cys for 5.75 and 4.5 hours, respectively.

Longer-chain thiols normally show the more negative desorption potentials. ${ }^{64}$ The $-0.96 \mathrm{~V}$ peak supports a model with a Cys bilayer with Cys molecules on top of each other, very likely through hydrogen bonding between carboxylate and ammonium groups. The excess charges of the two functional groups are partially compensated in the bilayer and would hamper liberation of the molecules into the solution. A similar model was proposed from observations of L-Cys adsorption on (polycrystalline) gold by surface plasmon resonance (SPR) spectroscopy. ${ }^{32}$ High-resolution in situ STM, cf. below further supports this view.

\subsection{Chronopotentiometry}

As observed by real time SPR monitoring, adsorption of Cys on polycrystalline gold is a fast process that depends on the solution L-Cys concentration. ${ }^{32} \mathrm{We}$ studied the dynamics of Cys adsorption on $\mathrm{Au}(100)$ by chronopotentiometry that records the time-dependent Nernstian potential change for redox systems in homogenous solution or/and immobilized on an electrode surface at sub-second time resolution. ${ }^{54,64}$ Among the functional groups in Cys, thiol is a reducing group and lowers the Nernstian potential.

The basis of the chronopotentiometric analysis is that a Cys molecule is first (physi)adsorbed. Adsorption of cysteinate anion is unlikely as this leads to etching of the Au-surface. ${ }^{24,25,65,66}$ There is obviously electron exchange between Cys and the gold electrode as noted by the potential drop. Physisorption is followed by conversion of the $\mathrm{Au}-\mathrm{S}$ unit through several stages to the final $\mathrm{Au}(0)-\mathrm{S}(0) \bullet$ form. As conversion progresses, more and more surface $\mathrm{Au}(0)-\mathrm{S}(0)$ • is formed. With a high and constant Cys concentration in the solution, this leads to gradual Nernstian potential increase in the equilibrium,

$$
\mathrm{R}-\mathrm{SH}_{\mathrm{ads}} € \mathrm{R}-\mathrm{S}_{\mathrm{ads}} \mathrm{g}+1 / 2 \mathrm{H}_{2}
$$


Chronopotentiometry requires that the electron exchange between adsorbed Cys species and the electrode is fast compared to all the conversion steps towards final equilibrium between thiylbound adsorbate and the electrode, so that Nernstian equilibrium is established throughout. With this understanding, the intermediate states in the Cys adsorption process are effectively time evolving ("quasi-") equilibrium states. This is known from other cases where the time dependent concentration of an electrochemically active species involved in separate, nonelectrochemical processes is followed by monitoring the Nernst potential of the species, and the kinetics of the non-electrochemical process determined. ${ }^{67,68}$ Figure 3 shows chronopotentiograms at different Cys concentrations and electrodes. When Cys is added to the solution, the $\mathrm{Au}(100)$ potential drops "instantly" by 300-600 $\mathrm{mV}$, followed by increase up to 30 minutes and stabilizing after 60 minutes, Figure 3A. The potential drop and subsequent increase both depend on the Cys concentration, higher Cys concentrations giving lower steady state potentials. Her potential drop is caused by adsorption of the Cys molecule, i.e. with sulfur in the low-oxidation thiolate state. This is followed by conversion to strong Au-S interaction, leading eventually to $\mathrm{Au}(0)-\mathrm{S}(0)$ gold-thiyl monolayer formation, i.e. with bound sulfur now in a higher formal oxidation state and therefore towards more positive Nernstian equilibrium potentials.

Cys adsorption on the three low-index gold substrates, $\mathrm{Au}(111), \mathrm{Au}(100)$ and $\mathrm{Au}(111)$ shows similar but distinct tendencies at given Cys concentration, Figure 3B and S2, suggesting that the details of Cys adsorption on the three surfaces are different. The graphite electrodes EPG and BPG were used as reference, as Cys does not chemisorb on these surfaces. Therefore potential drop was hardly observed when Cys solution was added, Figure 3C.
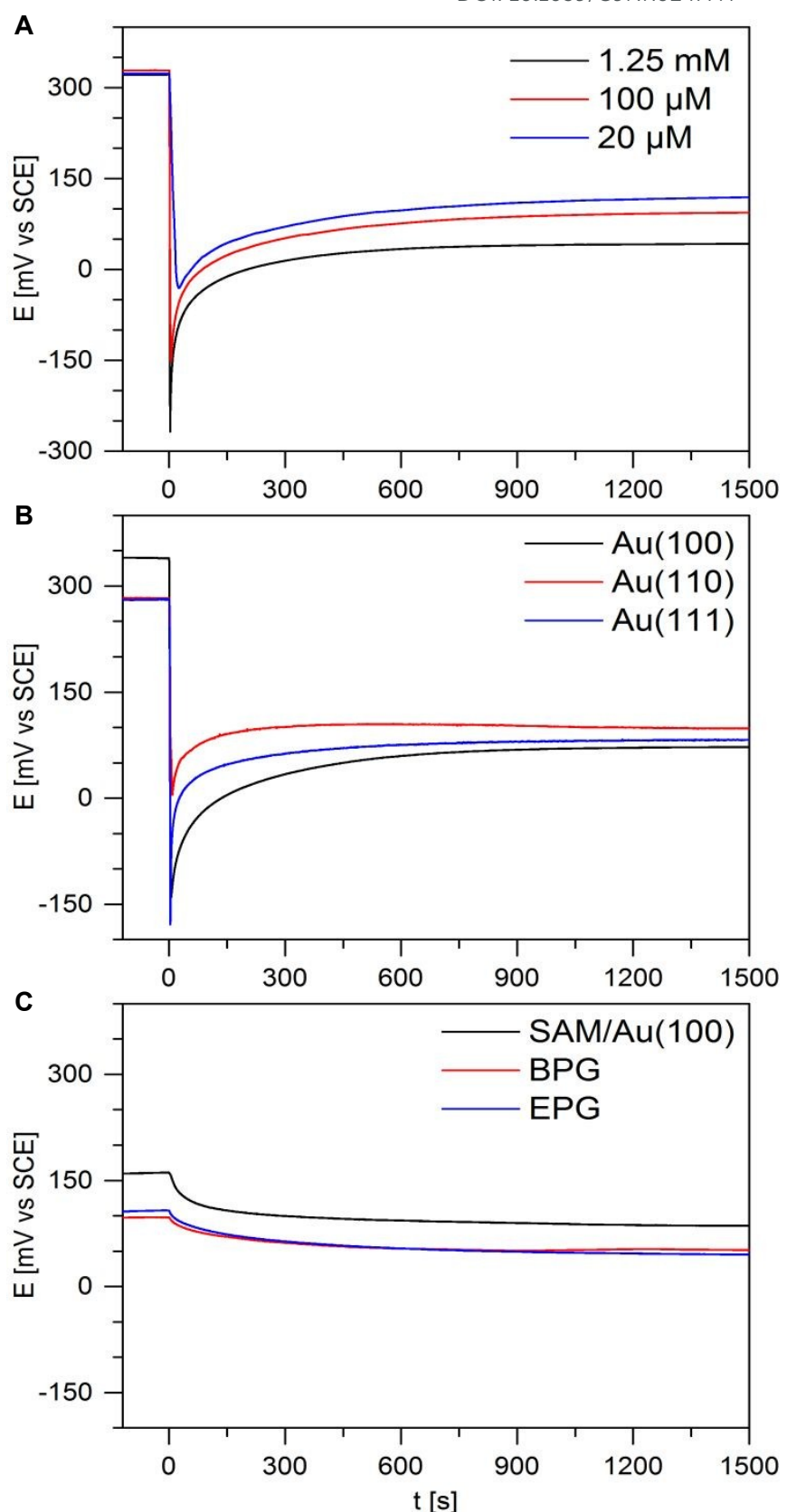

Figure 3. Chronopotentiometry of L-Cys adsorption in $25 \mathrm{mM}$ $\mathrm{KH}_{2} \mathrm{PO}_{4}(\mathrm{pH}$ 4.66). A $0.2 \mathrm{~mL}$ concentrated L-Cys solution is injected at $\mathrm{t}=0$. (A) Adsorption on $\mathrm{Au}(100)$ at different L-Cys concentrations. (B) Adsorption on single-crystal gold substrates using $250 \mu \mathrm{M}$ L-Cys. (C) Adsorption on inert substrates, i.e. a LCys adlayer on $\mathrm{Au}(100)$ (black), basal-plane graphite (red) and edge-plane graphite (blue) using $250 \mu \mathrm{M}$ L-Cys.

A small potential drop $(50 \mathrm{mV})$ was, however, still found for graphite electrodes as well as for Cys covered Au(100), Figure 3C suggesting that Cys in solution contributes an additional small potential change, while the main potential changes in Figure 3 are caused by Cys-Au chemisorption. The chronopotentiometry data could be represented as a multi-step process. 
Molecular details and intermediate adsorbed species will be addressed in Sections 3.3 and 3.4. The data display approximately exponential time evolution for all the three surfaces, with a minimum of two phases. In a two-phase view the time constants (adsorption rate constants) differ by roughly an order of magnitude. The time constants are similar for $\mathrm{Au}(100)$ and $\mathrm{Au}(111)$, but smaller for $\mathrm{Au}(110)$. Both time constants decrease with increasing Cys concentration. The amplitudes of the fast component are similar for all the $\mathrm{Au}$ electrodes, but the slow amplitude for $\mathrm{Au}(100)$ is twice that of $\mathrm{Au}(110)$ and $\mathrm{Au}(111)$ suggesting that this component has contributions from a process occurring solely on $\mathrm{Au}(100)$. The fast component amplitude increases weakly with increasing Cys concentration, while the slow component amplitude is independent of concentration. Bi-phasic time evolution was also found by SPR on polycrystalline $\mathrm{Au}$, where the two phases were assigned to bound Cys and a Cys bilayer. ${ }^{14}$ The chronopotentiometric data are summarized in Table 1.

Table 1. Biexponential time constants $t$ and amplitudes $A$, for chronopotentiometric Cys adsorption.

\begin{tabular}{cccccc}
\hline Electrode & $\begin{array}{c}\mathrm{c}_{\text {cys }} \\
{[\mathrm{mM}]}\end{array}$ & $\begin{array}{c}\mathrm{t}_{\text {fast }} \\
{[\mathrm{s}]}\end{array}$ & $\begin{array}{c}\mathrm{A}_{\text {fast }} \\
{[\mathrm{mV}]}\end{array}$ & $\begin{array}{c}\mathrm{t}_{\text {slow }} \\
{[\mathrm{s}]}\end{array}$ & $\begin{array}{c}\mathrm{A}_{\text {slow }} \\
{[\mathrm{mV}]}\end{array}$ \\
\hline $\mathrm{Au}(100)$ & 0.02 & 52 & -87 & 400 & -110 \\
$\mathrm{Au}(100)$ & 0.10 & 34 & -110 & 330 & -110 \\
$\mathrm{Au}(100)$ & 0.25 & 30 & -100 & 270 & -120 \\
$\mathrm{Au}(100)$ & 1.25 & 20 & -140 & 220 & -110 \\
$\mathrm{Au}(110)$ & 0.25 & 13 & -90 & 130 & -62 \\
$\mathrm{Au}(111)$ & 0.25 & 27 & -81 & 270 & -60 \\
$\mathrm{SAM} / \mathrm{Au}(110)$ & 0.25 & 44 & 43 & 530 & 27 \\
$\mathrm{BPG}$ & 0.25 & 15 & 10 & 230 & 36 \\
$\mathrm{EPG}$ & 0.25 & 62 & 18 & 430 & 39 \\
\hline
\end{tabular}

The simplest, two-phase kinetic scheme, with a single intermediate species, is represented as

$$
A_{r e d}^{\text {sol }} \underset{k_{-1}}{\stackrel{k_{1}}{\rightleftarrows}} A_{o x, i}^{a d s} \underset{k_{-2}}{\stackrel{k_{2}}{\rightleftarrows}} A_{o x, f}^{a d s}
$$

$A_{\text {red }}^{\text {sol }}$ is cysteine in solution, $A_{o x, f}^{a d s}$ the adsorbed final Au-thiyl species, ${ }^{24,25,65,66}$ and $A_{o x, i}^{\text {ads }}$ an intermediate species. $k_{1}, k_{-1}, k_{2}$, and $k_{-2}$ are the rate constants. Eq.(2) can represent different intermediate species, e.g. N-bound Cys or Cys dimer species
The nature of these cannot be identified from chronopotentiometry, but we have identified likely candidates by DFT calculations on differently oriented solvated Cys monomers and dimers on a $\mathrm{Au}(100)$ model surface, Section 3.4. Eq.(2) gives biexponential evolution of the coverage of the final adsorbed Au-thiyl Cys species, $\theta_{f}(\mathrm{t})$

$\theta_{f}(t)=\theta_{f}^{\infty}\left[1+\frac{R_{2}}{R_{1}-R_{2}} e^{R_{1} t}-\frac{R_{1}}{R_{1}-R_{2}} e^{R_{2} t}\right]$

with the two phases (rate constants)

$$
\begin{aligned}
& R_{1}=-\frac{1}{2}\left(k_{-1}+k_{-2}+k_{2}+k_{1}\left[A_{r e d}^{\text {sol }}\right]\right)+ \\
& \sqrt{\frac{1}{4}\left(k_{-1}+k_{-2}+k_{2}+k_{1}\left[A_{r e d}^{\text {sol }}\right]\right)^{2}-\left(k_{1} k_{-2}+k_{1} k_{2}\right)\left[A_{\text {red }}^{\text {sol }}\right]-k_{-1} k_{-2}}
\end{aligned}
$$

$R_{2}=-\frac{1}{2}\left(k_{-1}+k_{-2}+k_{2}+k_{1}\left[A_{\text {red }}^{\text {sol }}\right]\right)-$

$\sqrt{\frac{1}{4}\left(k_{-1}+k_{-2}+k_{2}+k_{1}\left[A_{\text {red }}^{\text {sol }}\right]\right)^{2}-\left(k_{1} k_{-2}+k_{1} k_{2}\right)\left[A_{\text {red }}^{\text {sol }}\right]-k_{-1} k_{-2}}$

$R_{1}$ and $R_{2}$ reduce to the rate constants of the forward steps, when the reverse reactions are disregarded, $R_{1} \rightarrow-k_{1}\left[A_{\text {red }}^{\text {sol }}\right], R_{2} \rightarrow-k_{2}\left(R_{1}, R_{2}<\right.$ $0)$. The equilibrium final state coverage is

$$
\theta_{f}^{\infty}=\frac{k_{1} k_{2}\left[\text { Asol }\left.\right|_{\text {red }} ^{\text {sol }}\right]}{R_{1} R_{2}}=\frac{k_{1} k_{2}\left[A_{\text {red }}^{\text {sol }}\right]}{\left(k_{1} k_{-2}+k_{1} k_{2}\left[A_{\text {red }}^{\text {sol }}\right]\right)-k_{-1} k_{-2}}
$$

The recorded quantity is not, however, directly the surface coverage (as in $\mathrm{SPR}^{32}$ ) but the reduction potential $E-E_{0}$, where $E_{0}$ is the standard equilibrium potential. Assuming first that $E-E_{0}$ is determined solely by the final state Cys thiyl chemisorption, $E-E_{0}$ is given by

$$
\begin{aligned}
& E-E_{0}=\frac{R T}{F} \ln \left(\frac{O x}{\operatorname{Re} d}\right)=\frac{R T}{F} \ln \frac{\theta(t)}{\left[A_{\text {red }}^{\text {sol }}\right]}=59(\mathrm{mV}) \log \frac{\theta_{f}^{\infty}}{\left[A_{\text {red }}^{\text {sol }}\right]} \\
& +59(\mathrm{mV}) \frac{R T}{F} \log \left[1+\frac{R_{2}}{R_{1}-R_{2}} e^{R_{1} t}-\frac{R_{1}}{R_{1}-R_{2}} e^{R_{2} t}\right]
\end{aligned}
$$


We then notice, first, that the recorded $\left(\mathrm{E}-\mathrm{E}_{0}\right) / \mathrm{t}$ correlations approach (bi)exponential but approach (bi)exponential behavior, when $\left|R_{1}\right| t>1$ and $\left|R_{2}\right| t>1$. Since

$\left|\frac{R_{1}}{R_{1}-R_{2}}\right|<<1$ and $\left|\frac{R_{2}}{R_{1}-R_{2}}\right| \approx 1$

there are two approximately exponential regimes, with a slow phase

$E-E_{0} \approx 59(\mathrm{mV}) \log \frac{\theta_{f}^{\infty}}{\left[A_{\text {red }}^{\text {sol }}\right]}+59(\mathrm{mV}) \frac{R T}{F}\left|\frac{R_{2}}{R_{1}-R_{2}}\right| \exp ^{-\left|R_{1}\right| t}$

when $\left|R_{1} /\left(R_{1}-R_{2}\right)\right| e^{\left|R_{2}\right| t}<1$, and a fast phase

$E-E_{0} \approx 59(m V) \log \frac{\theta_{f}^{\infty}}{\left[A_{\text {red }}^{\text {sol }}\right]}+59(m V) \frac{R T}{F}\left|\frac{R_{1}}{R_{1}-R_{2}}\right| \exp ^{-\left|R_{2}\right| t}$

when $\left|R_{2} /\left(R_{2}-R_{1}\right)\right| e^{|R| t}<1$, enabling determination of the rate constant combinations $\mathrm{R}_{1}$ and $\mathrm{R}_{2}$.

Secondly, $R_{1}+R_{2}$ and $R_{1} \times R_{2}$ reduce to

$$
\begin{aligned}
& R_{1}+R_{2}=-\left(k_{-1}+k_{-2}+k_{2}+k_{1}\left[A_{\text {red }}^{\text {sol }}\right]\right) \\
& R_{1} \times R_{2}=\left(k_{1} k_{-2}+k_{1} k_{2}\right)\left[A_{\text {red }}^{\text {sol }}\right]-k_{-1} k_{-2}
\end{aligned}
$$

The data do in fact follow this $\left[A_{\text {red }}^{\text {sol }}\right]$ dependence, Figure S3. The slopes and intercepts enable determining the rate constants $k_{1}, k_{-1}, k_{2}$, and $k_{-2}$ as $1.8 \mathrm{~s}^{-1}, 0.011 \mathrm{~s}^{-1}, 4 \times 10^{-3} \mathrm{~s}^{-1}$, and $5 \times 10^{-4} \mathrm{~s}^{-1}$, respectively. These values reflect the $E-E_{0}$ time evolution from Figure 3, with a fast initial phase where Cys is physisorbed followed by significantly slower conversion to the final, thiylbound Cys chemisorbed state, both steps shifted in favour of the products. In the following two sections we address the nature of the intermediate state(s).

However, the view of the Nernstian potential as determined solely by Cys thiyl adsorption is not compatible with all the data. The amplitudes of both phases are roughly the same, Table 1, but eqs.(3)-(10) would assign a significantly smaller amplitude, $\left|R_{1} /\left(R_{1}-R_{2}\right)\right| \ll<$ to the fast phase, $R_{2}$ than the amplitude, $\left|R_{2} /\left(R_{1}-R_{2}\right)\right| \approx 1$ assigned to the slow phase, $R_{1}$. This must be caused by a potential response not only to final state thiyl adsorption of Cys but also to intermediate adsorption. While eqs.(3)-(5) maintain validity, intermediate state potential response must therefore be included in a more complete description of the potential, eqs.(8)-(11). The surface coverage of the intermediate state, $\theta_{i}(t)$ also displays biexponential time evolution, i.e.

$$
\begin{aligned}
& \theta(t)=\theta_{f}^{\infty}\left[1-\frac{R_{2}}{R_{1}-R_{2}}\left(1+\frac{R_{1}}{k_{-2}}\right) e^{R_{2} t}+\frac{R_{1}}{R_{1}-R_{2}}\left(1+\frac{R_{2}}{k_{-2}}\right) e^{R_{1} t}\right] \\
& \theta_{i}^{\infty} \rightarrow \theta_{i}^{\infty}=\frac{k_{1} k_{-2}\left[A_{\text {red }}^{\text {sol }}\right]}{\left(k_{1} k_{-2}+k_{1} k_{2}\left[A_{\text {red }}^{\text {sol }}\right]-k_{-1} k_{-2}\right.}
\end{aligned}
$$

As $k_{-2}$ is a reverse rate constant an 11 19mall, both amplitudes are approximately $1+\left(R_{1} R_{2} / k_{-2}\right)$. Biexponential time evolution of the potential with significantly different phases but approximately equal amplitudes can thus be understood, if both the final state thiyl Cys adsorption and an intermediate, $\mathrm{N}$ bound Cys or S-bound bilayer contributes to the potential.

\subsection{In situ STM}

\subsubsection{Bare Au(100) and L-Cysteine on $\mathrm{Au}(100)$}

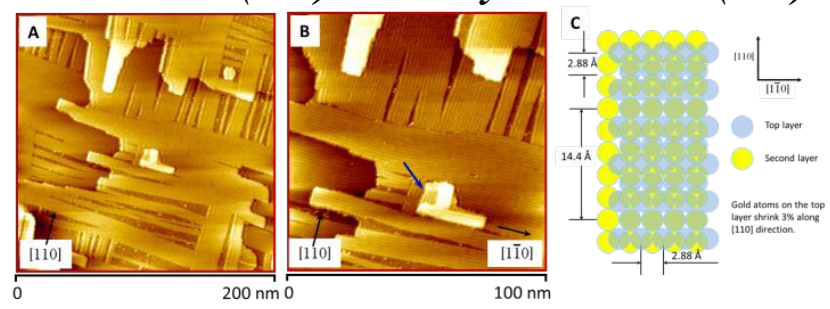

Figure 4. In situ STM images (A and $\mathrm{B}$ ) of bare $\mathrm{Au}(100)$ in 25 $\mathrm{mM} \mathrm{KH} \mathrm{PO}_{4}(\mathrm{pH} 4.7) . \mathrm{I}_{\mathrm{t}}=0.05 \mathrm{nA}, \mathrm{V}_{\text {bias }}=-0.01 \mathrm{~V} . \mathrm{E}_{\mathrm{W}}=-0.3 \mathrm{~V}$ vs $\mathrm{SCE}(\mathrm{C})$ is model of $\mathrm{Au}(100)(5 \times 20)$ reconstruction.

Cys monolayers on $\mathrm{Au}(100)$ were mapped by in situ STM. Bare $\mathrm{Au}(100)$ shows a clean surface from -0.30 to $+0.25 \mathrm{~V}$ vs SCE, with many highly 
ordered stripes at a periodic distance $1.40 \pm 0.2$ $\mathrm{nm}$, Figure 4A and B. Stripes on the same terrace are perpendicular to each other, visible even on small terraces, as indicated by a blue arrow in Figure 4B. This feature accords with $(5 \times 20)$ reconstruction, Figure $4 \mathrm{C}$ (as also seen in the $\mathrm{CV}$, Figure 1), with a square pattern of gold atoms in the second layer and a hexagonal pattern in the top layer. $(5 \times 20)$ reconstruction was also found for $\mathrm{Au}(100)$ in vacuum. ${ }^{62}$ Other groups have reported the $(1 \times 1) \mathrm{Au}(100)$ structure at more positive potentials. ${ }^{62,69,70}$

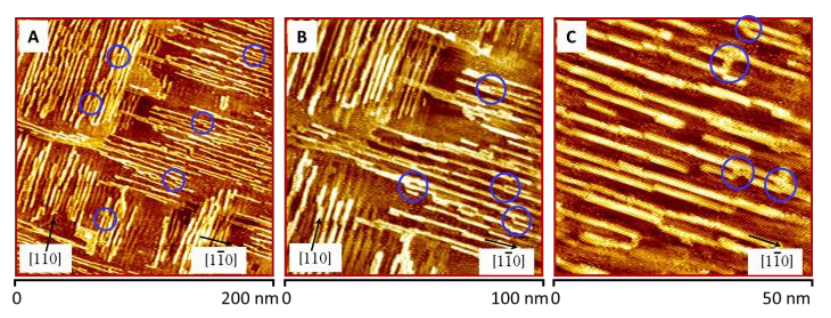

Figure 5. In situ STM images of L-Cys on $\mathrm{Au}(100) . \mathrm{I}_{\mathrm{t}}=0.2 \mathrm{nA}$, $\mathrm{V}_{\text {bias }}=-0.4 \mathrm{~V} . \mathrm{E}_{\mathrm{W}}=-0.1 \mathrm{~V}$ vs SCE. $200 \mu \mathrm{M}$ L-Cys in $25 \mathrm{mM}$ $\mathrm{KH}_{2} \mathrm{PO}_{4}(\mathrm{pH} 4.66)$.

In the presence of L-Cys, the $(5 \times 20)$ reconstruction is completely lifted and $\mathrm{Au}(100)$ totally covered by L-Cys SAMs (Figure 5), as for chemical thiol (thiyl) adsorption on $\mathrm{Au}(111)$ and $\mathrm{Au}(110){ }^{71-74}$ Cys monolayer adsorption on $\mathrm{Au}(100)$ does not accord with physical adsorption, such as for metalloporphyrin monolayers, for which both the adsorbed porphyrin monolayer and reconstructed substrate underneath are visible. ${ }^{70,75}$ New stripe-like features along both [110] and [1]̄0] directions following exactly the atomic rows of the $\mathrm{Au}(100)$ substrate underneath and parallel to the terrace steps appear on Cys adsorption, Figure 5A-C. The length of the stripes ranges from a few nm to several hundred $\mathrm{nm}$, and the distance between the stripes from 2-3 to 6-10 nm, depending on the adsorption conditions such as soaking time, Cys concentration, and potential. Low and dense coverage of stripes is found at short (3.5 hours, $0.06 \mathrm{mM}$ Cys) and long adsorption times (20 hours, $0.5 \mathrm{mM}$ Cys), respectively, Figure S4.

Notably, both stripes and the space between the stripes are covered by Cys monolayers, Figure S5. Strong STM image contrasts are caused by high electronic conductivity, Section 3.4. These stripes are very different from the well-defined stripes for alkane or branched chiral thiolates on $\mathrm{Au}(111){ }^{76-78}$ The most conspicuous features are: (1) 'Fork'-like features among the stripes, indicated by blue circles in Figure 5. Such a feature is not found in L-Cys monolayers on $\mathrm{Au}(111)$ or $\mathrm{Au}(110)$, and is unexpected and highly notable. Possible reasons will be addressed in Sections 3.4 and 4.
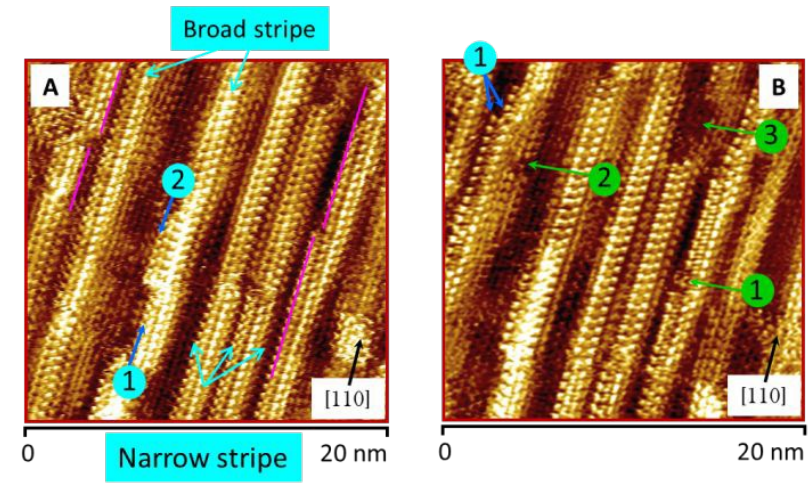

Figure 6. In situ STM images of L-Cys on $\mathrm{Au}(100)$. $\mathrm{I}_{\mathrm{t}}=0.15 \mathrm{nA}$, $\mathrm{V}_{\text {bias }}=-0.25 \mathrm{~V}$. $\mathrm{E}_{\mathrm{W}}=-0.15 \mathrm{~V}$ vs. SCE. $200 \mu \mathrm{M}$ L-Cys in $25 \mathrm{mM}$ $\mathrm{KH}_{2} \mathrm{PO}_{4}$ (pH4.66). (A) Mismatch (red lines and blue circles). (B) Individual defects (green circles 1 and 2 ) and defect patch (green 3 ) inside stripes; individual defects between stripes (blue circle 1).

(2) Parallel stripes with two widths, in highly ordered units packed along $\mathrm{Au}(100)$ atomic rows underneath. As indicated by blue arrows (Figure $6 \mathrm{~A})$, broad $(3.2 \pm 0.2 \mathrm{~nm})$ and narrow $(2.0 \pm 0.1$ nm) stripes co-exist in L-Cys SAMs.

(3) Mismatch along molecular rows is found in both broad and narrow stripes, as encircled by blue lines in circle 1 and 2 and marked by red lines, respectively, Figure 6A.

(4) Individual defects between stripes (blue circle 1 ), inside the narrow (green circle 1) and broad (green 2) stripes, along with defect patches or domains (green circle 3), Figure 6B.

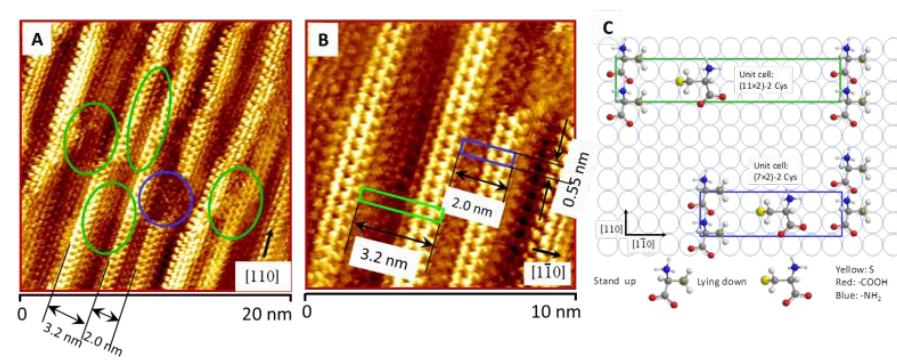

Figure 7. (A) and (B) in situ STM images of L-Cys on Au(100). $\mathrm{I}_{\mathrm{t}}$ $=0.15 \mathrm{nA}, \mathrm{V}_{\text {bias }}=-0.25 \mathrm{~V} . \mathrm{E}_{\mathrm{W}}=-0.15 \mathrm{~V}$ vs SCE. $200 \mu \mathrm{M} \mathrm{L}-$ cysteine in $25 \mathrm{mM} \mathrm{KH} \mathrm{PO}_{4}$ (pH4.66). (A) Interaction among molecules between the stripes, and molecules in a stripe with other molecules in a defect patch are highlighted with green 
circles, and blue circle respectively. (B) Unit cells are overlaid on the image. (C) Tentative adsorption model with $(11 \times 2)-2$ Cys (green) and (7×2)-2 Cys (blue) unit cell. L-Cys molecules in both planar (flat or lying down) and upright (stand up) orientation are suggested.

(5) Orientation of molecules among the stripes (green circles), and stripes with defect patches (blue circle), suggesting strong network interactions, Figure 7A.

(6) The closest periodic distance is the same, 0.55 $\mathrm{nm}$ in either broad or narrow stripes, i.e. twice the gold atom diameter underneath. The $3.2 \mathrm{~nm}$ and $2.0 \mathrm{~nm}$ distances correspond to 11 and 7 gold atoms in perpendicular direction. Unit cells for broad and narrow stripes are therefore described as $(11 \times 2)-2$ Cys and $(7 \times 2)-2$ Cys, Figure $7 \mathrm{~B}$.

Models with tentative structures for such unit cells are shown in Figure 7C and Figure S6, resting on both upright and recumbent Cys orientations in both broad and narrow stripes. Mixture of strong and dark contrast in STM images found in 1-butanethiol SAMs on $\mathrm{Au}(111)$ was caused by upright and laying down orientation respectively. ${ }^{18}$ it is reasonable that vertical Cys here gives strong contrast than planar Cys due to facile tunnelling route. Coadsorption of water molecules is likely due to the wide space in the broad stripes $((11 \times 2)-2 \mathrm{Cys})$, but not included in the model. Adsorption of a second Cys molecule on top of the upright Cys molecule is possible e.g. by formation of a $\mathrm{H}-$ bonded dimer perpendicular to the $\mathrm{Au}$ (100) surface, Section 3.4. More detailed insight is provided in Section 3.4 based on quantum chemical modelling and MC simulations, leading to more precise accordance between the modelling and the data.

The in situ STM data match the L-Cys coverage from electrochemical reductive desorption, Figure 2 . The unit cells $(11 \times 2)-2$ Cys and $(7 \times 2)-2$ Cys give 92 and $58.2 \AA^{2}$ per L-cysteine molecule, corresponding to $1.81 \times 10^{-10}$ and $2.86 \times 10^{-10}$ $\mathrm{mol} / \mathrm{cm}^{2}$, respectively. The average coverage of Cys on $\mathrm{Au}(100)$ from reductive desorption is $2.52( \pm 0.15) \times 10^{-10} \mathrm{~mol} / \mathrm{cm}^{2}$, i.e. $68 \AA^{2}$ per Lcysteine molecule, suggesting a mixture of $(7 \times 2)$ 2 Cys and $(11 \times 2)-2$ Cys domains. The model with two Cys molecular orientations implies two sets of close lying reductive desorption peaks, consistent with the double peak at $0.67 \mathrm{~V}$ vs SCE in Figure 2. Moreover, a vertical Cys dimer would require a stronger reductive desorption force than monomeric Cys (recumbent), suggesting that the $-0.96 \mathrm{~V}$ peak, Figure 2 is assigned to Cys dimer.

\subsubsection{D-Cys and L, D-Cys adlayers on Au(100)}

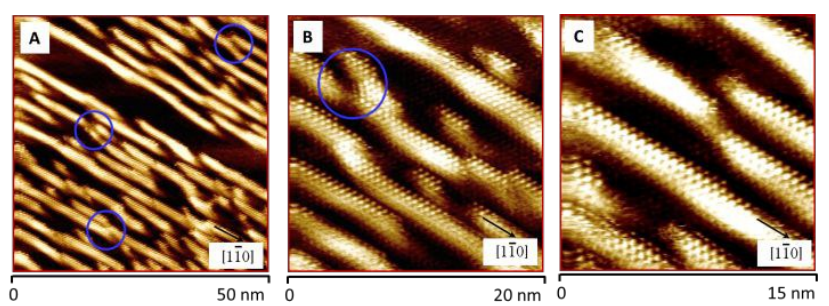

Figure 8. In situ STM images of D-Cys on $\mathrm{Au}(100)$. $\mathrm{I}_{\mathrm{t}}=0.56 \mathrm{nA}$, $\mathrm{V}_{\text {bias }}=-0.15 \mathrm{~V}, \mathrm{E}_{\mathrm{W}}=-0.40 \mathrm{~V}$ vs SCE. $200 \mu \mathrm{M}$ D-Cys in $25 \mathrm{mM}$ $\mathrm{KH}_{2} \mathrm{PO}_{4}(\mathrm{pH} 4.66)$.

Monolayers of pure D-Cys and 1:1 racemic D,LCys on $\mathrm{Au}(100)$ were also investigated by in situ STM. Pure L-Cys, D-Cys and D,L-Cys are indistinguishable at the macroscopic level, but clearly different at the nanoscale level of in situ STM. Similar to L-cysteine, both D-cysteine and D,L-cysteine adlayers are composed of uniform stripes with characteristic 'forks', indicated by blue circles in Figure 8 and 9. The main observable difference between the three groups of enantiomers is that while L-and D-Cys give closely similar images, a larger number of nanoscale defects are found in the D,L-Cys adlayers, Figure 9. The defects are either solitary or appear in groups inside the stripes or on either side of the stripes. Defects are caused by molecular mismatching during self-assembly, which points to the importance either of hydrogen bonding or spatial incompatibility among the different Cys molecular enantiomers in the D,L-Cys SAMs. Similar observations were encountered recently for high-resolution in situ STM images of the chiral 2-butanethiols ${ }^{76,78} \mathrm{We}$ shall return to this issue in Section 4. 


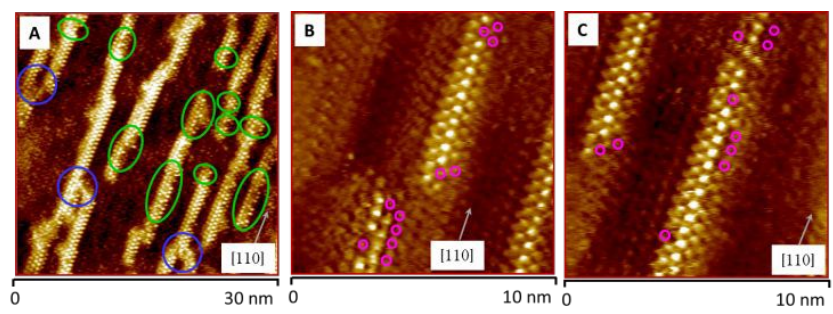

Figure 9. In situ STM images of L,D-Cys on $\mathrm{Au}(100)$. (A) $\mathrm{I}_{\mathrm{t}}=$ $0.12 \mathrm{nA}, \mathrm{V}_{\text {bias }}=-0.20 \mathrm{~V}, \mathrm{E}_{\mathrm{W}}=-0.15 \mathrm{~V}$ vs SCE, (B) and (C) $\mathrm{I}_{\mathrm{t}}=2.40 \mathrm{nA}, \mathrm{V}_{\text {bias }}=-0.20 \mathrm{~V}, \mathrm{E}_{\mathrm{W}}=-0.20 \mathrm{~V}$ vs SCE. $100 \mu \mathrm{M}$ L-Cys and $100 \mu \mathrm{M}$ D-Cys in $25 \mathrm{mM} \mathrm{KH}_{2} \mathrm{PO}_{4}$ (pH4.66). Defects within stripes (green circles), in fork-like structures (blue circles) and individual missing molecules (purple circles) are highlighted.

\subsection{Molecular Modelling}

\subsubsection{Cys monomer and dimer adsorption on Au(100)}

The adsorption energy of a Cys molecule from solution was found to be small (bond energy $0.19 \mathrm{eV})$ and comparable to the adsorption energy of a water molecule on $\mathrm{Au}(100)$ (-0.3 $\left.\mathrm{eV}^{79}\right)$. This can be attributed to physisorption where intermolecular interactions (H-bonds, van der Waals forces) dominate. Chemisorption accompanied by $\mathrm{S}-\mathrm{H}$ bond cleavage therefore entirely dominates the overall adsorption process, eq. (1). The reaction product is an adsorbed Cys radical $\mathrm{R}-\mathrm{S}_{\mathrm{ads}} \bullet$ strongly bound to the $\mathrm{Au}$ surface through the sulfur atom. We modelled the first step, i.e. $\mathrm{R}-\mathrm{SH}_{\mathrm{ads}} \in \mathrm{R}-\mathrm{S}_{\mathrm{ads}} \bullet+\mathrm{H}_{\mathrm{ads}}$, by DFT calculations.
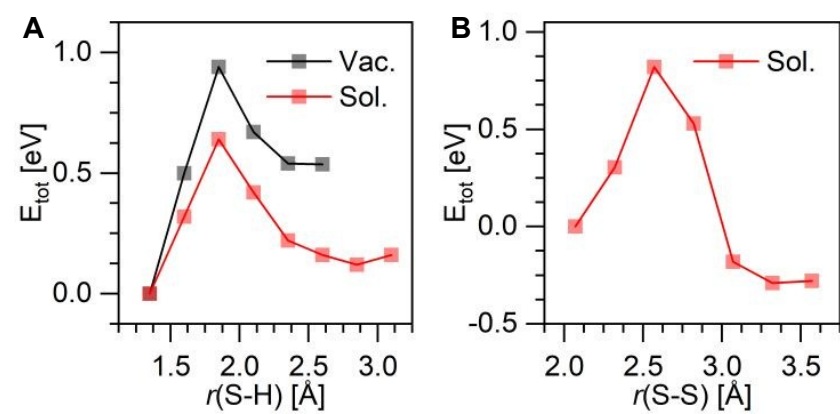

Figure 10. Potential energy surfaces describing (A) breaking of the S-H bond on dissociative adsorption of an L-Cys molecule at the $\mathrm{Au}(100)$ surface (the total energy calculated from the deepest value), and (B) the S-S bond cleavage on dissociative adsorption of an L-Cystine molecule at the Au(100) surface (the total energy of the initial point taken as zero).

The S-H bond was scanned with steps of $0.25 \AA$, while the rest of the Cys molecule was fully optimized. Representative results are shown in Figures 10A and S7. The energy profiles disclose high barriers, both for the metal/vacuum interface $(0.94 \mathrm{ev})$, and with correction for aqueous environment $(0.64 \mathrm{eV})$. Chemisorption is therefore likely to be slow. Both overall processes were found to be endothermic: +0.69 $\mathrm{eV}$ (vacuum) and $+0.27 \mathrm{eV}$ (solution) largely due to the $\mathrm{S}-\mathrm{H}$ bond breaking step. Additional energy gain comes, however, from the formation of a $\mathrm{H}_{2}$ molecule by the recombination of the adsorbed $\mathrm{H}$-atoms. We therefore conclude that reaction (1) is feasible, with an estimated reaction heat of $-0.26 \mathrm{eV}$. Simulations of the same process on the $\mathrm{Au}(111) /$ water interface using a combination of DFT and Reactive Classic Molecular Dynamics reported recently ${ }^{39}$ gave an estimated energy barrier of $0.3 \mathrm{eV}$, substantiating the exothermic character of the overall process.

We investigated also the dissociative adsorption of a cystine molecule (R-S-S-R) at the Au(100) surface, including solvation effects similarly to process (1), Figures $10 \mathrm{~B}$ and S8. The energy profile, Figure $10 \mathrm{~B}$, is exothermic but with a notable barrier $(0.82 \mathrm{eV})$. The entropic factor also favors dissociative adsorption. The reverse process, i.e. recombination, $2 \mathrm{R}-\mathrm{S}_{\mathrm{ads}} €$ R-S-S-R $\mathrm{R}_{\mathrm{ads}}$ on $\mathrm{Au}(100)$ is therefore both thermodynamically and kinetically unlikely.

Cys dimers turn out to be stable Cys species in both DFT and Monte Carlo simulations, see below. In what follows we therefore take the formation of Cys dimers from the adsorbed products of eq. (1) as an important initial step of the SAM formation on $\mathrm{Au}(100)$. With a further view on recent observations of the adsorption of other chiral thiol molecules ${ }^{76-78,80}$ the most stable dimer combinations of L- and D-Cys in all the three stereo-selective combinations, L,L, D,D and L,D were addressed. The most stable radical forms of these species include cyclic dimers, where two Cys radicals are contacted through two hydrogen bonds -O---HO-. The most stable dimer for Cys radicals in zwitterionic form has two -COO--- $\mathrm{H}_{3} \mathrm{~N}$ - contacts.

We define the energy of dimer formation on the $\mathrm{Au}(100)$-surface as:

$$
\Delta E_{f}^{\operatorname{dim}}=\Delta E_{a d s}(C y s \operatorname{dim})-2 \Delta E_{a d s}(C y s)
$$


where $\Delta E_{a d s}(C y s)$ and $\Delta E_{a d s}(C y s \mathrm{dim})$ are the adsorption energies of a single monomeric Cys species and a cysteine dimer, respectively. Further,

$$
\begin{aligned}
& \Delta E_{a d s}(C y s)=E_{t o t}\left(A u_{n}-C y s\right)-E_{t o t}\left(A u_{n}\right)-E_{t o t}(C y s) \\
& \Delta E_{a d s}(C y s \operatorname{dim})= \\
& E_{t o t}\left(A u_{n}-C y s \operatorname{dim}\right)-E_{t o t}\left(A u_{n}\right)-2 E_{t o t}(C y s)
\end{aligned}
$$

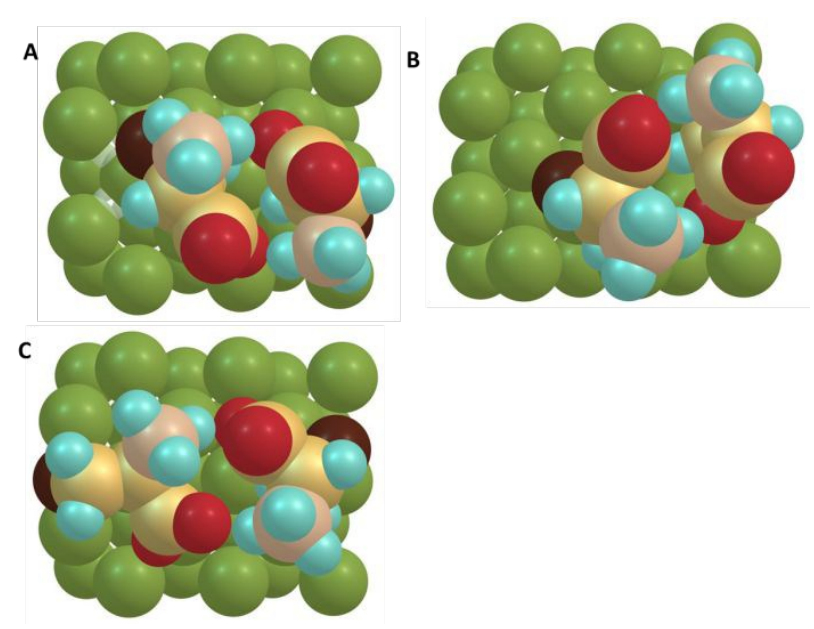

Figure 11. Optimized orientation of three different $\mathrm{R}_{\mathrm{Z}}-\mathrm{R}_{\mathrm{Z}}$ dimers adsorbed at the $\mathrm{Au}(100)$ surface in planar (horizontal) orientation; L,L (A); D,D (B); L,D (C).

For brevity, we will refer to thiols as ' $M$ ', and thiyl radicals as ' $R$ ' with a subscript ' $Z$ ' to indicate zwitterionic forms. Some results are compiled in Tables S1-S4 and Figures S9-S11. The data in Table $\mathrm{S} 1$ do not describe real electrochemical adsorption from the solution, but these results are needed for calculations using eq. (14) and (16). As seen from Tables S1-S4, formation of all Cys dimers at the $\mathrm{Au}(100)$ surface is feasible. The deepest $\Delta E_{f}^{\mathrm{dim}}$ values (from $-3.6 \mathrm{eV}$ to $-2.7 \mathrm{eV}$ ) were found for the $\mathrm{R}_{\mathrm{Z}}-\mathrm{R}_{\mathrm{Z}}$ dimers (Figure 11, Table S3). Formation of $\mathrm{R}-\mathrm{R}$ dimers is less likely. The corresponding $\Delta E_{f}^{\mathrm{dim}}$ values (ranging from $-1 \mathrm{eV}$ to $-0.65 \mathrm{eV}$ ) accord with those reported by Chapman and associates $^{36}$ on the L,L (-1.04 eV) and L,D (-1.14 $\mathrm{eV})$ dimers adsorbed at the $\mathrm{Au}(110) /$ vacuum interface. The charge of the adsorbed Cys dimers was found to be small ranging from -0.05 to -0.23 $e^{-}$. The chirality of the Cys species significantly affects both the adsorption energy and the geometry of the dimers, Tables S2, S3. $\Delta E_{f}^{\mathrm{dim}}$ for the $\mathrm{R}_{\mathrm{Z}}-\mathrm{R}_{\mathrm{Z}}$ dimers, increases in the order $\mathrm{L}, \mathrm{L}<$ $\mathrm{L}, \mathrm{D}<\mathrm{D}, \mathrm{D}$, while a different sequence is observed for the R-R dimers, D,D $<\mathrm{L}, \mathrm{D}<\mathrm{L}$, L. The adsorption energy of different Cys forms and their dimers with the metal surface thus depends on the interaction of the sulfide-, amino-, and carboxyl groups with the metal surface. In turn, the position and orientation of these molecular groups strongly depend on the geometry of the Cys dimers, which differs noticeably for different enantiomer pairs. This can explain qualitatively the observed trends.

We addressed furthermore two different types of "vertical" Cys dimer. The first form was composed of a Cys radical on the metal surface bound to a Cys molecule from the solution, i.e. ('R-M'). In the second case, the dimer consists of a zwitterionic radical at the metal surface bound to a zwitterionic molecule from the solution side (' $\mathrm{R}_{\mathrm{Z}}-\mathrm{M}_{\mathrm{Z}}$ '). Our estimations show that the former structure is not feasible, whereas the second one is stable (Figure 12, Table S4). The formation energy of these dimers is in a narrow range from $-1.15 \mathrm{eV}$ to $-1.05 \mathrm{eV}$ and slightly chirally sensitive. The normal dipole moment projections calculated for the $\mathrm{Au}_{\mathrm{n}}-\mathrm{Cys}$ vertical dimer are small and in the range $0 .-1.72 \mathrm{D}$. This might favour additionally the growth of rows composed of these dimers.

The formation energy of a L-Cys R-M dimer in planar orientation (Figure S11) was estimated to be $-0.51 \mathrm{eV}$. This type of Cys dimer plays the role of an intermediate which might impede SAM formation. Reaction (1) is thus crucial in the SAM formation, but may proceed slowly because of steric immobility of bound Cys molecules. This might stop further initial growth of rows from Cys radicals and favor the appearance of "stripes" clearly observed by in situ STM. 


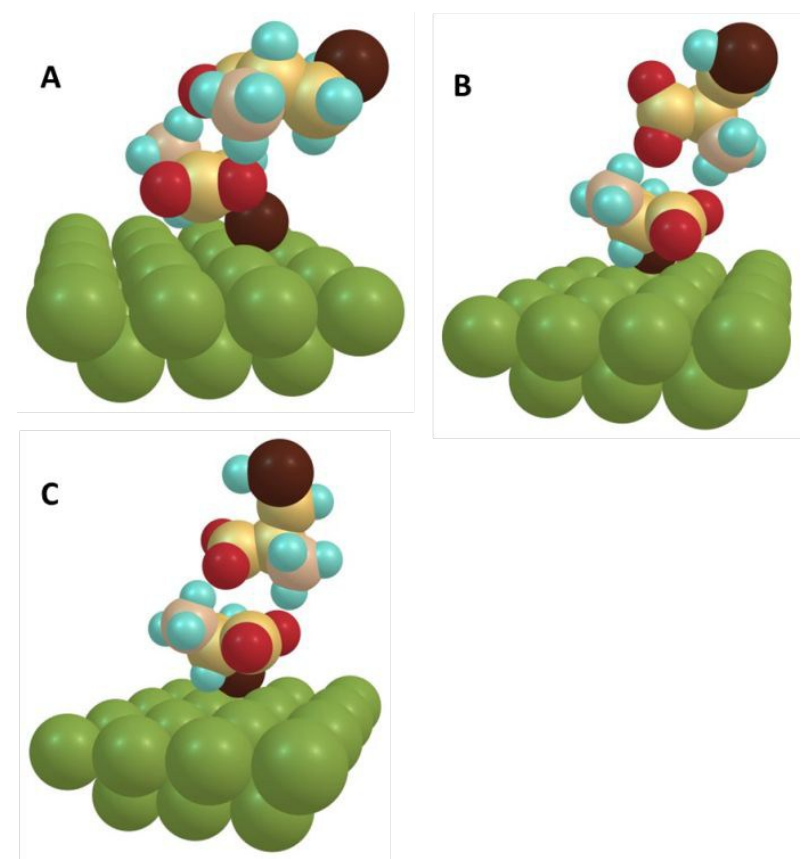

Figure 12. Optimized structures of three Different $R_{Z}-M_{Z}$ dimers on $\mathrm{Au}(100)$ in vertical orientation; (A) L,L; (B) D,D; (C) L,D. $R_{Z}$ is adsorbed at the metal surface, while $\mathrm{M}_{\mathrm{Z}}$ is located on top (from the solution side).

The STM contrasts were calculated for all adsorbed Cys zwitterionic dimers considered. The resonance integral $\left(V_{\text {if }}\right)$ values were around $10^{-5}$ Hartree which corresponds to the diabatic (non-adiabatic) limit of electron transfer. ${ }^{24,25}$ As seen from Figures 13-14, the STM images for different types of the adsorbed species are different. "Stereoselectivity" of the STM contrasts is predicted as well. Distinct submolecular resolution of the images (which reveal two or three lobes) is observed for the L,L vertical and L,D planar dimers. In the model images for the adsorbed vertical dimers, the isosurface height corresponding to the brightest areas $\left(h_{\max }\right)$ ranges from $8.5 \AA$ to $11 \AA$, which is within the 6.4 to $10 \AA$ range for the zwitterionic Cys dimers in planar orientation. Although $h_{\max }$ depends on the $V_{\text {if }}$ value which is a variable parameter, it can thus be argued that the STM contrasts related to the vertical Cys zwitterionic dimers give the strongest STM contrast.

The following sequence of events can then be proposed from the DFT calculations, Section 3.4.2:
A
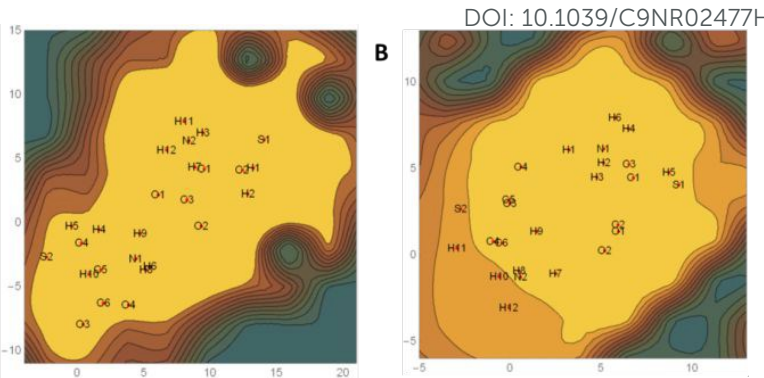

c

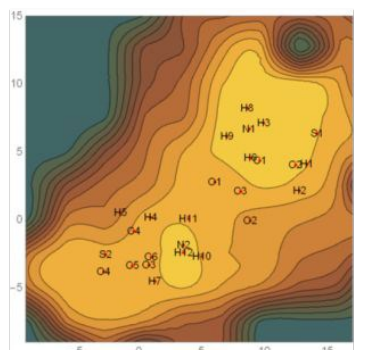

Figure 13. STM contrasts computed for $\mathrm{R}_{\mathrm{Z}}-\mathrm{R}_{\mathrm{Z}}$ dimers adsorbed on $\mathrm{Au}(100)$ in planar (horizontal) orientation; (A) L,L; (B) D,D; (C) L,D. Distances in a.u.

\section{First step}

Adsorption of Cys molecules, R-SH at the $\mathrm{Au}(100)$-surface from the bulk solution. As this adsorption bond is weak, fast establishment of adsorption $€$ desorption equilibrium is expected. Adsorption of Cys zwitterions $\left(\mathrm{R}^{+,-}-\mathrm{SH}\right)$ in molecular form is less likely due to strong hydration effects, even though this species prevails in the aqueous solution. Adsorbed Cys radicals R-S. and $\mathrm{H}$ atoms appear next due to $\mathrm{S}-\mathrm{H}$ bond dissociation; R-S. forms in turn an adsorbed Cys zwitterion radical $\mathrm{R}^{+,-}-\mathrm{S}$. by fast intramolecular reorganization. This is likely to dominate the first chronopotentiometric step.

\section{Second step}

This step is the formation of a second Cys (zwitterion) monolayer on top of the first, leading to differently composed and oriented Cys molecular bilayers. These are 
dominated by dimers of adsorbed zwitterion radical $\mathrm{R}^{+,-}-\mathrm{S}$. adjacent to the electrode surface hydrogen bonded to a zwitterion molecular form towards the solution, in horizontal or upright orientation $\left({ }^{\prime} \mathrm{R}_{\mathrm{Z}}-\mathrm{M}_{\mathrm{Z}}\right.$ '). Dimer formation is still incomplete and the adlayer composed of a mixed mono- and bilayer. The DFT computations give brighter STM contrasts for the upright than for the horizontal Cys dimer orientation. Different adsorbed intermediate (R-R and R-M) dimer forms might exist as well and affect the dynamics of the SAM formation.

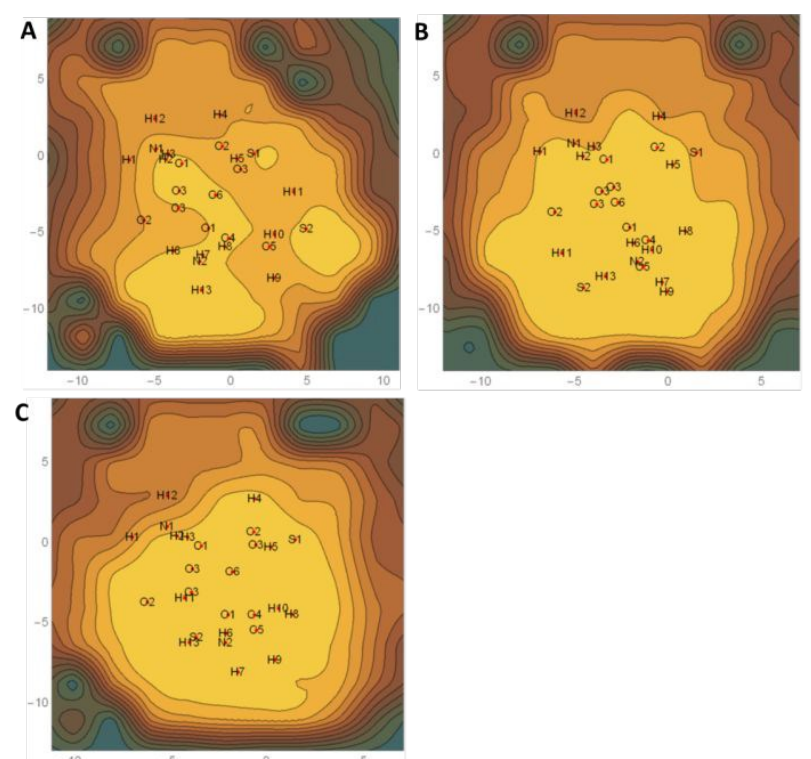

Figure 14. STM contrasts computed for three $R_{Z}-M_{Z}$ dimers adsorbed on $\mathrm{Au}(100)$ in vertical orientation; (A) L,L; (B) D,D; (C) L,D. Distances in a.u.

\section{Third step}

The first and second steps are proposed to lead to formation of a dense zwitterion Cys mixed mono- and bilayer. From reported voltammetric and in situ STM studies of the time evolution of cysteamine ${ }^{72}$ and propanethiol monolayers ${ }^{73}$ on $\mathrm{Au}(111)$ electrode surfaces, the primary adlayers, however, evolve through several disordered phases towards the ultimate highly ordered adlayers. In the present study these phases would include transition from disordered, vertically or horizontally oriented zwitterionic Cys monolayers and Cys radical/molecular dimer combinations. These act further as directional nucleation sites into the highly ordered surface rows and stripes observed by in situ STM. This step may be what is observed as the slow chronopotentiometric phase.

\subsubsection{MC simulation of Cys adlayer formation on $\mathrm{Au}(100)$}

Some results of kinetic MC simulations for the squared $100 \times 100$ surface site lattice illustrating four different stages of the Cys adlayer formation are sampled and shown in Figure 15 , where $(A)-(D)$ correspond to different numbers of simulation steps, see Table 2.

Table 2. Fractions of Cys species and structures in the adlayer on $\mathrm{Au}(100)$ estimated for different stages of the monolayer formation based on kinetic MC simulations. Stages and corresponding number of steps (x104): (A) 2.25, (B) 9, (C) 27.5, (D) 225. $\mathrm{M}=$ molecule, $\mathrm{R}=$ radical, $\mathrm{R}_{\mathrm{Z}}=$ zwitter ion radical, $\mathrm{M}_{\mathrm{Z}}=$ zwitter ion molecule.

\begin{tabular}{lllllll}
\hline Stage & $\mathbf{M}$ & $\mathbf{R}$ & $\mathbf{R}_{\mathbf{Z}}$ & $\begin{array}{l}\mathbf{R}-\mathbf{M} \\
\text { dimer }\end{array}$ & $\begin{array}{l}\mathbf{R}_{\mathbf{Z}}-\mathbf{R}_{\mathbf{Z}} \\
\text { planar } \\
\text { arrays }\end{array}$ & $\begin{array}{l}{\left[\mathbf{R}_{\mathbf{Z}}-\mathbf{M}_{\mathbf{Z}}\right]_{\mathbf{n}}} \\
\text { vertical rows }\end{array}$ \\
\hline$(A)$ & 0.86 & 0.11 & 0. & 0.03 & 0. & 0. \\
$(B)$ & 0.08 & 0.02 & 0.14 & 0.01 & 0.7 & 0.05 \\
(C) & 0.01 & 0. & 0.03 & 0. & 0.82 & 0.14 \\
(D) & 0. & 0. & 0.01 & 0. & 0.75 & 0.24 \\
\hline
\end{tabular}


At the initial stage of adlayer formation, $(A)$, adsorbed Cys molecules, $M$, clearly dominate. There are also small amounts of Cys radicals, R, and R-M dimers. The number of molecules is then drastically reduced, and rows of adsorbed zwitterionic forms (planar and vertical) appear at the intermediate stage $(B)$. Adsorbed single Cys zwitterions, $\mathrm{R}_{\mathrm{Z}}$, and a very small amount of $\mathrm{R}-\mathrm{R}$ and $\mathrm{R}-\mathrm{M}$ dimer is also seen. In the next stages, $(C)$ and $(D)$, approaching the final state we observe further growth of rows of vertical Cys dimers, some of which show a branched structure, similar to the "forked" stripes observed by in situ STM. The R-R and R-M dimers have here practically disappeared. Rows of zwitterions in planar orientation, $\mathrm{R}_{\mathrm{Z}}-\mathrm{R}_{\mathrm{Z}}$, tend to form arrays in all the stages. Data on the fractions of different Cys species and structures on $\mathrm{Au}(100)$ are collected in Table 2.
A
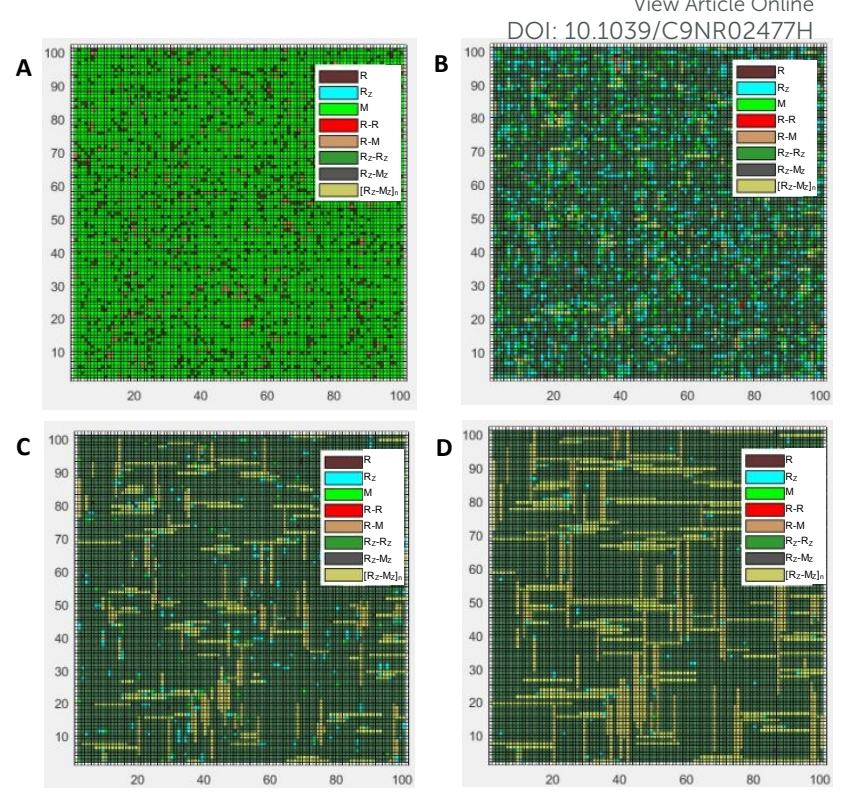

Figure 15. 2D-pictures illustrating four different stages of the formation of a Cys adlayer on $\mathrm{Au}(100)$ built using the data of kinetic MC simulations, cf. Table 2. M, R, $\mathrm{R}_{\mathrm{Z}}$ are Cys molecules, radicals, and radical zwitter ions, respectively. $R-R, R-M, R_{Z}-R_{Z}$, $\mathrm{R}_{\mathrm{Z}}-\mathrm{M}_{\mathrm{Z}}$ are dimer species. $\left[\mathrm{R}_{\mathrm{Z}}-\mathrm{M}_{\mathrm{Z}}\right]_{\mathrm{n}}$ indicates rows of vertical dimers.

The events that emerge from the MC calculations match broadly the voltammetric, chronopotentiometric, and in situ STM data. The dominating final state is represented by rows of vertically oriented zwitterion dimers, $\left[\mathrm{R}_{\mathrm{Z}}-\mathrm{M}_{\mathrm{Z}}\right]_{\mathrm{n}}$, but with a number of "fork-like" "irregularities". The initial fast chronopotentiometric phase is likely to incorporate stages $(A)$ and $(B)$, possibly also partly $(C)$, as these stages cannot be separated. The slow chronopotentiometric phase is likely dominated by stages (C) and $(D)$, i.e. the growth and formation of $\left[\mathrm{R}_{\mathrm{Z}}-\mathrm{M}_{\mathrm{Z}}\right]_{\mathrm{n}}$ from single-molecule zwitterionic forms or from differently oriented (planar) zwitterionic rows. $\left[\mathrm{R}_{\mathrm{Z}}-\mathrm{M}_{\mathrm{Z}}\right]_{\mathrm{n}}$ is most likely what dominates the in situ STM images. The abundant "forklike" structures and other surface structural 
irregularities together with the dual voltammetric reductive desorption peaks, however, also suggest that residual single zwitterionic molecules and planar versus vertically oriented zwitterionic dimers are abundant enough to contribute to both voltammetry and in situ STM.

For a more rigorous description of the structure and dynamics of molecular adlayers at metal electrodes in contact with electrolyte solution, periodical DFT calculations with CPMD or reactive molecular dynamics would be tempting. Among other advantages such methods can describe the solvent explicitly at the molecular level, which is another challenge for molecular modelling.

The combined approach based on the energy characteristics of different Cys species adsorbed on the $\mathrm{Au}(100)$ surface, model STM contrasts, and MC simulation points to the crucial role of the zwitterionic forms in different orientations in the final monolayer. Adsorbed zwitterions are also nucleation centers for the growth of rows of Cys zwitterions (and their dimers) in different directions and likely to be crucial in the initial and intermediate adlayer formation stages. The appearance of "vertical" $\mathrm{R}_{\mathrm{Z}}-\mathrm{M}_{\mathrm{Z}}$ dimers is favored additionally, as the second Cys zwitterionic molecule enters from the solution bulk with constant high concentration of this species.

The unique organization of Cys dimers in "stripes" and "forks" on the $\mathrm{Au}(100)$ surface compared with Cys adsorption on $\mathrm{Au}(111)$ and $\mathrm{Au}(110)$ where only a single Cys surface species/orientation prevails is at first unexpected. The overall surface adlayer organization is, however, determined by a detailed interplay between lateral intermolecular forces (hydrogen bonds, van der Waals interactions) and the Ausurface structure. The square Au-atomic $\mathrm{Au}(100)$ surface is in fact well suited for unhindered Cys organization in "stripes" along the surface atomic rows, where the dimers are better nucleation centres than the monomers, and which would also trigger further organization in square Cys lattices, Figure 5 and Figure 15. The hexagonal $\mathrm{Au}(111)$ structure is much less compatible with "stripe" organization and Cys forms instead highly ordered monolayers with $(\sqrt{ } 3 \times \sqrt{ } 3) \mathrm{R} 30^{\circ}$, $(3 \sqrt{ } 3 \times 6) \mathrm{R} 30^{\circ}$, and $(4 \times \sqrt{7}) \mathrm{R} 19^{\circ}$ cluster-like networks depending on supporting electrolyte and $\mathrm{pH}$ as noted. ${ }^{16,18,19,31}$. The missing row $\mathrm{Au}(110)$ structure is clearly visible in highresolution in situ STM imaging ${ }^{25}$ and might have been expected also to be a favourable template for "stripe" adlayer organization, but such a surface structure seems not to be competitive with the observed highly ordered $\mathrm{c}(2 \times 2)$ dense monolayer with two Cys molecules in each unit cell. A recent study of Cys adsorption on polycrystalline gold based on surface plasmon resonance spectroscopy in fact also came to the conclusion that Cys dimers are formed, ${ }^{32}$ emphasizing again the importance of the local surface structure in the formation of Cys adlayers.

Kinetic MC simulations can shed light on this intriguing observation. As a reference for Cys adlayer formation on $\mathrm{Au}(100)$, MC simulations were carried out for $\mathrm{Au}(110)$ (Table S5-S6) illustrating the uniqueness of Cys adlayer formation at the $\mathrm{Au}(100)$ surface. Indeed, the results on $\mathrm{Au}(110)$ look quite different as compared with those obtained earlier for the $A u(100)$ surface. In the case of $A u(100)$, we see a competition between arrays of zwitter ions adsorbed in planar orientation with stripes formed by Cys dimers in vertical orientation (Table 2, Figure 
15). In contrast, for $A u(110)$ arrays of "planar" zwitter ions fully dominate at the final stage and the fraction of "vertical" dimers passes a maximum (see Table S6 and Figure S12-13). The latter finding qualitatively agrees with the $c(2 \times 2)$ lattice as disclosed by the experimental in situ STM data $^{25}$

It is important to note that the probabilities in Table S5 were introduced in a "phenomenological" way. To find them from first principles is equivalent to performing fully atomistic molecular dynamics simulations, which remain a complex and challenging problem.

Together the voltammetric, potentiometric, and in situ STM data, combined with the DFT and MC calculations thus offer a detailed and coherent view of Cys adlayer structures on electrochemical $\mathrm{Au}(100)$ electrode surfaces. This extends to the molecular mechanism with adsorption and nucleation directed growth of Cys adsorbate rows and the Cys monomer and dimer intermediates.

\section{Conclusion and Perspectives}

As an electrochemical core target, adsorption of the amino acid Cys on singlecrystal and polycrystalline Au-electrode surfaces has long been in focus of electrochemical surface science. In the present work we report a comprehensive study of the high-resolution Cys adlayer structure on electrochemical $A u(100)$ electrode surfaces as well as dynamic features of the adsorption process. Electrochemical high-resolution in situ STM of Cys adsorption on single-crystal Au(111)and $\mathrm{Au}(110)$-electrode surfaces have been reported before, but very little attention given neither to the third of the low-index surfaces, $\mathrm{Au}(100)$, nor to a comparison between the three enantiomeric L-, D-, and racemic L,Dforms, which are the targets of the present study. Cys adsorption dynamics on electrochemical single-crystal Au-electrode surfaces has not been addressed before either. At the same time, the high-resolution structure of the Cys adlayers on the $\mathrm{Au}(100)$ electrode surface has disclosed intriguing features not seen for the other low-index surfaces.

We have used an experimental approach which combines voltammetry, chronopotentiometry, and electrochemical in situ STM to single-molecule resolution. The experimental approach was supported and guided by both DFT and MC calculations, to map how the adsorbed solvated Cys species are organized on the $A u(100)$-electrode surface. Such a combined experimental and theoretical study of the adsorption of solvated enantiomeric chiral Cys monomers and dimers has not been reported before.

The approach used has led to a coherent view of the structure and mechanism of Cys 
adlayer formation on the Au-electrode surface. CV displays two reductive desorption peaks instead of the single peak on $\mathrm{Au}(111)^{18}$ and $\mathrm{Au}(110)$ electrode surfaces $^{25}$. One peak, at $\approx-0.7 \vee$ coincides approximately with the $\mathrm{Au}(111)$ and $\mathrm{Au}(110)$ peaks, while the second peak at $0.3 \mathrm{~V}$ more negative potential is unique to $\mathrm{Au}(100)$. The peak areas are significantly smaller than for $\mathrm{Au}(111)$, and the peak ratio about 1:2 with the $-0.7 \mathrm{~V}$ peak the smallest. This is a first indication that two adsorbed species co-exist on the $A u(100)$-surface. The peaks were tentatively assigned to Cys monomer and $\mathrm{H}$ bonded upright dimer species at $-0.7 \mathrm{~V}$ and $0.97 \mathrm{~V}$.

Dual-structure adlayers was supported by chronopotentiometry, using a generic twostep kinetic model. Chronopotentiometry appears to record signals both from the final, presumably dimeric adsorbed Au-S bound species, and from intermediate possibly monomeric Au-S bound states, but does not offer direct insight into the molecular nature of these (Cys monomer or dimer). Neither can other rapidly equilibrating intermediates such as Cys bound via the ammonium or carboxylate groups be excluded.

Cys adlayer structures on $\mathrm{Au}(111)$-electrode surfaces has been mapped to molecular resolution by in situ STM. The surface lattice structure depends sensitively on both electrolyte and $\mathrm{pH}$. Cys monolayers on $\mathrm{Au}(110)$ has also been mapped, even to submolecular resolution by in situ STM and show a $c(2 \times 2)-2$ lattice with two Cys in each unit cell, ${ }^{25}$ but no high-resolution imaging has been reported for Cys on $\mathrm{Au}(100)$-electrode surfaces. Neither has comparative in situ STM of the different enantiomeric Cys forms been reported.

Molecular scale in situ STM resolution in the present study discloses a unique stripe-like pattern of adsorbed Cys molecular species on the $A u(100)$-electrode surface, with additional "fork-like" structures and defects or domains of defects. The pure enantiomeric D- and L-forms display very similar surface structural patterns, whereas racemic L,D-Cys displays many more defects. Differences such as these were also reported for the two chiral 2-butanethiols among the four isomeric butanethiols. ${ }^{76-78}$

Enantiomer-specific molecular adsorption can be triggered by substrate Au-surface atoms around defect structures and step edges which can themselves be chiral, leading to different numbers of defects for different enantiomers of chiral adsorbate molecules. Au-surface atoms also become chiral, once adsorbate molecules are bound, and whole adsorbate superstructures 
(domains etc.) can be chiral for nonchiral molecular adsorbates and vice versa. Further detailed discussions are given elsewhere $34,76,78,81-83$. The in situ STM images show still another feature to support that the equilibrated final state surface Cys adlayer holds more than a single Cys species, as suggested by voltammetry and chronopotentiometry. Both the stripes and the space between the stripes accommodate adsorbed Cys molecules, with two Cys orientations, as reported for cysteamine adsorption on $\mathrm{Au}(111)^{72}$. Upright Cys orientation offers a site for adsorption of a second Cys molecule to form a Cys bilayer on the $A u(100)$-surface such as suggested by the in situ STM images and according with the observation of a second, more negative voltammetric potential peak.

A theoretical frame for the data was, finally provided. This frame included, first DFT calculations of the energetics and in situ STM contrasts of a variety of solvated monomers and dimers in different orientations as well as radical, zwitterion and molecular forms in different orientations on a model Au(100)-surface. Secondly, MC computations led to molecular understanding of the adsorption process with all the stripe and fork structural elements as well as intermediates in the adsorption process.
DFT was based on a cluster model for the $\mathrm{Au}(100)$-surface, with focus on the different Cys monomers and dimers in radical and zwitterion forms, and in planar or vertical orientation. In contrast to previous reports, ${ }^{33-}$ ${ }^{36}$ we also included the solvent environment. The results of the DFT modelling were used to calculate model STM contrasts, and chiral effects on adsorption of L- and D-Cys molecules. The quantum chemical results combined with a kinetic MC scheme offer a direct impression of the ultimate adlayer structure as well as of crucial stages in the adlayer formation process. This approach was an immediate support and guide for the in situ STM images and the chronopotentiometric data.

Based on the totality of experimental data, and the quantum chemical DFT and MC calculations, we have suggested a sequence of molecular events in the Cys/Au(100) adsorption process. The overarching mechanistic view is that a Cys radical monolayer dominated by the zwitterionic form is formed at first, followed by adsorption of a second layer. The latter is first a disordered bilayer but reorganizes slowly to the final ordered row and stripe adlayer.

As a final observation, the stripe-like in situ Cys surface bilayer structure is only observed for $A u(100)$ and is quite different 
from the in situ Cys $\mathrm{c}(2 \times 2)$ monolayer on $\mathrm{Au}(110)^{25}$ and the various monolayer structures on $\mathrm{Au}(111)^{18,26}$. Reasons for this difference cannot be precisely identified, but both the DFT and MC calculations in the present report point to the crucial role of zwitterionic dimers unique to $\mathrm{Au}(100)$, and both giving strong in situ STM contrasts and acting as nucleation sites for full adlayer formation. The anisotropic charge distribution of a zwitterionic dimer, further supported by the atomic rows of $\mathrm{Au}$-atoms in the square lattice of $A u(100)$ could induce corresponding anisotropic further directional bilayer growth ultimately seen as stripes in the in situ STM images and the MC calculations. As noted, stripe-like surface structures of the Cys adlayer are not seen on the $A u(111)-$ and $A u(110)$-surfaces. It was argued in Section 3.4 that this is associated with the overall surface adlayer organization being determined by a detailed interplay between lateral intermolecular forces and Cys interactions with the quite different $\mathrm{Au}$ surface structures, emphasizing the importance of the local surface structure in the formation of Cys adlayers.

Associated content

\section{Supporting information.}

Further characterization of reference experiments and descriptions of experimental setups are given in the supporting information. This material is available free of charge via the Internet at http://pubs.acs.org.

\section{Author Information \\ Corresponding Author jz@kemi.dtu.dk.}

ORCID:

Christian Engelbrekt: 0000-0003-3679-3666

Renat R. Nazmutdinov: 0000-0003-4827-2649

Dmitrii Glukhov: 0000-0003-0797-9066

Jiawei Yan: 0000-0002-0045-6169

Bingwei Mao: 0000-0002-9015-0162

Jens Ulstrup: 0000-0002-2601-7906

Jingdong Zhang: 0000-0002-0889-7057

Notes

The authors declare no competing financial interest.

\section{Acknowledgement}

Financial support from the Lundbeck Foundation (R69-A8097) and Independent Research Fund Denmark (DFF 4093-00297) to J. Z., Independent Research Fund Denmark (DFF 5054-00107) to CE, the Russian Science Foundation (project № 17-13-01274) to R.R.N., T.T.Z. and J.U; The National Science Foundation of China (2016J01075) to JY, The National Science Foundation of China (21533006) BM., and ELECTRONANOMAT (Grant Agreement Number: PIRSES-GA-2012-318990) under European Framework Programme-Marie Curie Actions to J. U. is acknowledged.

\section{References}

1. Paulsen, C. E.; Carroll, K. S. Cysteinemediated Redox Signaling: Chemistry, Biology and Tools for Discovery, Chem. Rev., 2013, 113, 4633 - 4679.

2. Poole, L. B. The Basics of Thiols and Cysteines in Redox Biology and Chemistry, Free Radical Biology and Medicine, 2015, 80, 148157. 
3. Ralph, T. R.; Hitchman, M. L.; Millington, J. P.; Walsh, F. C. The Electrochemistry of LCystine and L-Cysteine part 2: Electrosynthesis of L-Cysteine at solid electrodes, J. Electroanal. Chem., 1994, 375, 17-27.

4. Seselj, N.; Engelbrekt, C.; Ding, Y.; Hjuler, H. A.; Ulstrup, J.; Zhang, J. Tailored Electron Transfer Pathways in Aucore/Ptshell-graphene Nanocatalysts for Fuel Cells. Adv. Energy Mater. 2018, 8, Art. No. 170260912 pp.

5. Honda, M.; Baba, Y.; Sekiguchi, T.; Shimoyama, I.; Hirao, N. Electrochemical Immobilization of Biomolecules on Gold Surface Modified with Monolayered LCysteine. Thin Solid Films, 2014, 556, 307310.

6. Jocelyn, P. C. The Standard Redox Potential of Cysteine-Cystine from the Thiol-Disulphide Exchange Reaction with Glutathione and Lipoic Acid, European J. Biochem., 1967, 2, 327-331.

7. Zagal, J. H.; Herrera, P. Electrochemistry of Cysteine and Cystine on Metal-Phthalocyanines Adsorbed on a Graphite Electrode, Electrochimica Acta, 1985, 30, 449-454.

8. Ralph, T. R.; Hitchman, M. L.; Millington, J. P.; Walsh, F. C. The Electrochemistry of LCystine and L-Cysteine part 1: Thermodynamic and Kinetic Studies, J. Electroanal. Chem., 1994, 375, 1-15.

9. Fawcett, W. R.; Fedurco, M.; Kováčová, Z.; Borkowska, Z. Electrochemistry of Cysteine on Low-Index Single-Crystal Gold Electrodes, Langmuir, 1994, 10, 912-919.

10. Chen, S. M. The Electrocatalytic Reactions of Cysteine and Cystine by Water-soluble iron Porphyrin, Manganese Porphyrin and Iron (II) Phenanthrolines, Electrochimica Acta, 1997, 42, 1663-1673.

11. Song, F.; Briseno, A. L.; Zhou, F. Redox Reactions of an Transformation between Cysteine-Mercury Thiolate and Cystine in Metallothioneins Adsorbed at thin Mercury Film Electrode, Langmuir, 2001, 17, 4081-4089.

12. Dourado, A. H. B.; Batista, A. P. de L., Oliveira-Filho, Sumodjo, P. T. A.; Torresi. S. I.
C. de. L-Cysteine Electrooxidation in Alkaline and Acidic Media: a Combined Spectroelectrochemical and Computational Study, RSC Advances, 2017, 7, 7492-7501.

13. Zhang, J.; Christensen, H. E. M.; Ooi, B. L.; Ulstrup, J. In situ STM Imaging and Direct Electrochemistry of Pyrococcus Furiosus Ferredoxin Assembled on Thiolate-Modified Au(111)- Surfaces, Langmuir, 2004, 20, 1020010207.

14. Patil, B., Kobayashi, Y.; Fujikawa, S.; Okajima, T. Direct Electrochemistry and Intramolecular Electron Transfer of Ascorbate Oxidase Confined on L-Cysteine Self-assembled Gold Electrode, Bioelectrochemistry, 2014, 95, $15-22$.

15. Tudös, A. J.; Johnson, D. C. Dissolution of Gold Electrodes in Alkaline Media Containing Cysteine, Anal. Chem., 1995, 67, 557-560.

16. Dakkouri, A. S.; Kolb, D. M.; EdelsteinShima, R.; Mandler, D. Scanning tunneling microscopy study of L-cysteine on $\mathrm{Au}(111)$, Langmuir, 1996, 12, 2849-2852.

17. Dodero, G.; De Michieli, L.; Caveller, O.; Rolandi, R.; Oliveri, L.; Dacca, A.; Parodi, R. Lcysteine chemisorption on gold: an XPS and STM study, Colloids and Surfaces $A$ Physicochemical and Engineering Aspects, 2000, 175, 121-128.

18. Zhang, J.; Chi, Q.; Nielsen, J. U.; Friis, E. P.; Andersen J. E. T.; Ulstrup J. TwoDimensional Cysteine and Cystine Cluster Networks on Au(111) Disclosed by Voltammetry and in Situ STM, Langmuir, 2000, 16, 7229 7237.

19. Xu, Q.; Wan, L.; Wang, C.; Bai, C.; Wang, Z.; Nozawa, T. New Structure of L-Cysteine Self-Assembled Monolayer on Au(111): Studies by in Situ Scanning Tunneling Microscopy, Langmuir, 2001, 17, 6203-6206.

20. Yang, W.; Gooding, J. J.; Hibbert, D.; Brynn, Characterization of Gold Electrode Modified with Self-assembled Monolayer of L-Cysteine for Adsorption Stripping Analysis of Copper, $J$. Electroanal. Chem., 2001, 516, 10-16.

21. Kühnle A.; Linderoth, T. R.; Hammer, B.; Besenbacher F. Chiral Recognition in Dimerization of Adsorbed Cysteine Observed by 
Scanning Tunneling Microscopy. Nature, 2002, 415, 891-893.

22. Kühnle, A.; Linderoth, T.R.; Besenbacher, F. Self-Assembly of Monodispersed, Chiral Nanoclusters of Cysteine on the $\mathrm{Au}(110)-(1 \times 2)$ Surface. J. Am. Chem. Soc., 2003, 125, 1468014681.

23. Kühnle, A.; Linderoth, T.R.; Besenbacher, F. Enantiospecific Adsorption of Cysteine at Chiral Kink Sites on $\mathrm{Au}(110)-(1 \times 2)$. J. Am. Chem. Soc., 2006, 128, 1076-1077.

24. Nazmutdinov, R. R.; Zhang, J.; Zinkicheva, T. T. ; Manyurov, I. R.; Ulstrup, J. Adsorption and In Situ Scanning Tunneling Microscopy of Cysteine on $\mathrm{Au}(111)$ : Structure, Energy and Tunneling Contrasts, Langmuir, 2006, 22, 75567567.

25. Zhang J.; Chi Q.; Nazmutdinov, R. R.; Zinkicheva T.; Bronshtein, M.D. Submolecular Electronic Mapping of Single Molecules by In Situ Scanning Tunneling Imaging, Langmuir, 2009, 25, 2232 - 2240.

26. Mateo-Marti, E.; Rogero, C.; Gonzalez, C.; Sobrado, J. M.; Andres, P. L. de; Martin-Gago, J. A. Interplay Between Fast Diffusion and Molecular Interaction in the Formation of SelfAssembled Nanostructures of S-Cysteine on $\mathrm{Au}(111)$, Langmuir, 2010, 26, 4113-4118.

27. Oliveira, F. C. M. de; Serrano, S. H. Electrochemically Active L-Cysteine Gold Modified Electrodes, Electrochimica Acta, 2014, 125, 566-572.

28. Di, J.; Hu, Y.; Song, Yan, Tu, Y. Formation of Hydrous Gold (I) Oxide in the Process of Selfassembled Cysteine on Gold Nanoparticles and its Electrocatalytic Application, J. of Electroanal. Chem., 2012, 674, 12-16.

29. Dourado, A.H. B.; Silva, R. A.; Torresi, R. M.; Sumodjo, P. T. A.; Arenz, M.; Torresi, S. I. C. de, Kinetics, Assembling, and Conformation Control of L-Cysteine Adsorption on $\mathrm{Pt}$ Investigated by in situ FTIR Spectroscopy and QCM-D, ChemPhysChem, 2018, 19, 2340-2348.

30. Chen, C. J. (Ed.), Introduction to Scanning Tunnelling Microscopy, Second Edition, 2008, Oxford University Press.

31. Zhang, J.; Welinder, A. C.; Chi, Q.; Ulstrup, J. Electrochemically Controlled Self-assembled
Monolayers Characterized with Molecular and Sub-molecular Resolution. Phys Chem Chem Phys., 2011, 13, 5526-5545.

32. Tawil, N.; Hatef, A.; Sacher, E.; Maisonneuve, M.; Gervais, T.; Mandeville, R.; Meunier, M. Surface Plasmon Resonance Determination of the Binding Mechanisms of LCysteine and Mercaptoundecanoic Acid on Gold, J. Phys. Chem. C, 2013, 117, 6712-6718.

33. Kühnle, A.; Molina, L.M. ; Linderoth, T.R. ; Hammer, B.; Besenbacher, F. Growth of Unidirectional Molecular Rows of Cysteine on $\mathrm{Au}(110)-(1 \times 2)$ Driven by Adsorption Induced Surface Rearrangements, Phys. Rev, Lett., 2004, 93, 086101-086104.

34. Fajín, J.L.C.; Gomes, J.R.B.; Cordeiro, M.N.D.S., DFT Study of the Adsorption of D-(L) Cysteine on Flat and Chiral Stepped Gold Surfaces, Langmuir, 2013, 29, 8856-8864.

35. Buimaga-Larinka, L.; Morari, C., Effect of Conformational Symmetry upon the Formation of Cysteine Clusters on the Au(110)-(1x1) Surface: A First Principle Study, J. Phys. Chem., 2013, 117, 20351-20360.

36. Chapman, C.R.L.; Ting, E.T.M.; Kereszti, A.; Paci, I., Self-Assembly of Cysteine Dimers at the Gold Surface: A Computational Study of Competing Interactions. J. Phys. Chem. C, 2013, 117, 19426-19435.

37. Höffling, B.; Ortmann, F.; Hannewald, K.; Bechstedt, Adsorption of Cysteine on the Au(110)-surface: A Density Functional Theory Study, High Performance Computing in Science and Engineering'09, (Eds.) Nagel W. E., Springer-Verlag Berlin Heidelberg, 2010.

38. Burimaga-larinca, L.; Morari, C. Adsorption of Cysteine Clusters on $\mathrm{Au}(110)-(1 \times 1)$ Surface: a DFT Study. RSC Adv., 2013, 3, 5036-5044.

39. Monti, S.; Carravetta, V.; Ågren. H. Simulation of Gold Functionalization with Cysteine by Reactive Molecular Dynamics, $J$. Phys. Chem. Lett., 2016, 7, 272-276.

40. Cuesta, A.; Kolb, D. M. The Structure of Bromide and Chloride Adlayers on $\mathrm{Au}(100)$ Electrodes: an in situ STM Study, Surface Science, 2000, 465, 310-316.

65. McDonald, O.; Cafolla, A. A.; Carty, D.; Sheerin, G.; Hughes, G. Photoemission, 
NEXAFS and STM Studies of Pentacene Thin Films on Au(100). Surface Science, 2006, 600, 3217-3225.

41. Labayen, M.; Magnussen, O. M. In situ Video-STM Study of the Potential-induced $(1 \times 1)$ $\rightarrow$ "hex" Transition on Au(100) Electrode Surfaces in $\mathrm{Cl}^{-}$Containing Solution. Surface Science, 2004, 573, 128-139.

42. Yan, J., Tang, Y.; Sun, C.; Su, Y.; Mao, B. STM Study on Nonionic Fluorosurfactant Zonyl FSN Self-assembly on $\mathrm{Au}(100)$ : (3/11/1) Molecular Lattice. Corrugations, and AdsorbateEnhanced Mobility. Langmuir, 2001, 17, 38293834.

43. Valenzuela-Benavides, J.; Herrera-Zaldivar, M. Structural Transitions of Chemisorbed Iodine on $\mathrm{Au}(100)$ : A STM and LEED Study. Surface Science, 2005, 592, 150-158.

44. Tang, Y.; Yan, J.; Zhu, F.; Sun, C.; Mao, B. Comparative Electrochemical Scanning Tunnelling Microscopy Study of Nonionic Fluorosurfactant Zonyl FSN Self-assembled Monolayers on $\mathrm{Au}(111)$ and $\mathrm{Au}(100)$ : a Potential-Induced Structural Transition. Langmuir, 2011, 27, 943-947.

45. Prado, C.; Prieto, F.; Rueda, M.; Feliu, J.; Aldaz, A. Adenine Adsorption on $\mathrm{Au}(111)$ and $\mathrm{Au}(100)$ Electrodes: Characterisation, Surface Reconstruction Effects and Thermodynamic Study. Electrochimica Acta, 2007, 52, 31683180 .

46. Randler, R. J.; Kolb, D. M.; Ocko, B. M.; Robinson, I. K. Electrochemical Copper Deposition on $\mathrm{Au}(100)$ : A Combined in situ STM and in situ Surface X-ray Diffraction Study. Surface Science, 2000, 447, 187-200.

47. Kolb, D. M.; Schneider, J. Surface Reconstruction in Electrochemistry: $\mathrm{Au}(100)$ (5x20), $\mathrm{Au}(111)-(1 \times 23)$ and $\mathrm{Au}(110)-(1 \times 2)$. Electrochimica Acta, 1986, 31, 929-936.

48. Schneider, J.; Kolb, D. M. Potential-induced Surface Reconstruction of Au(100), Surface Science, 1988, 193, 579-592.

49. Jiang, Y.; Liang, X.; Ren, S.; Chen, C.; Fan, L. The Growth of Sulfur Adlayers on
$\mathrm{Au}(100) . \quad J . \quad$ Chem. Phys., 2015, 142, 064708.

50. Hamelin, A. Cyclic Voltammetry at Gold Single-crystal Surfaces. Part 1. Behaviour at Low-index Faces, J. of Electroanal. Chem., 1996, 407, 1-11.

51. Engelbrekt, C.; Jensen, P. S.; Sørensen, K. S.; Ulstrup, J.; Zhang, J Complexity of Gold Nanoparticle Formation Disclosed by Dynamics Study, J. Phys. Chem. C, 2013, 117, 11818 11828.

52. Felice, R. D.; Selloni, A. Adsorption Modes of Cysteine on $\mathrm{Au}(111)$ : Thiolate, amino-thiolate, disulfide, J. Chem. Phys., 2004, 120, 4906-4914. 53. Zhao, Y., Zhou, F.; Zhou, H.; Su, H. The Structural and Bonding Evolution in Cysteinegold Cluster Complexs, Phys. Chem. Chem. Phys, 2013, 15, 1690-1698.

54. Nazmutdinov, R.R.; Shapnik, M.S. Contemporary quantum chemical modelling of electrified interfaces, Electrochim. Acta, 1996, 41, 2253-2263.

55. Frisch, M. J.; Trucks, G. W.; Schlegel, H.

B. et al.; Gaussian 09, Revision B.01, 2009,

Gaussian, Inc., Wallingford CT.

56. Hay, P.J.; Wadt, W.R. Ab initio effective core potentials for molecular calculations. Potentials from $\mathrm{K}$ to $\mathrm{Au}$ including the outermost core orbitals. J. Chem. Phys. 1985, 82, 270-283.

57. Chemcraft - graphical software for visualization of quantum chemistry computations. https://www.chemcraftprog.com 58. Salvatore P.; Nazmutdinov, R. R.; Ulstrup J.; Zhang, J. DNA bases assembled on the $\mathrm{Au}(110) /$ electrolyte interface: A Combined Experimental and Theoretical Study, J. Phys. Chem. B, 2015, 119, 3123-3134.

59. Cuesta, A.; Kleinert, M.; Kolb, D. M. The Adsorption of Sulfate and Phosphate on Au(111) and $\mathrm{Au}(100)$ Electrodes: an in situ STM Study. Phys. Chem. Chem. Phys, 2000, 2, 5684-5690.

60. Loglio, F.; Schweizer, M.; Kolb, D. M. In situ Characterization of Self-Assembled Butanethiol 
Monolayers on $\mathrm{Au}(100)$ Electrodes. Langmuir, 2003, 19, 830-834.

61. Q. Chi, J. Zhang, J. E. T. Andersen and J. Ulstrup, 'Ordered Assembly and Controlled Electron Transfer of the Blue Copper Protein Azurin at Gold(111) Single-Crystal Substrates. J. Phys. Chem. B, 2001,105, 4669-4679.

62. Yoshimoto, S.; Sawaguchi, T.; Mizutani, F.; Taniguchi, I. STM and Voltammetric Studies on the Structure of a 4-pyridinethiolate Monolayer Chemisorbed on $\mathrm{Au}(100)-(1 \times 1)$ Surface. Electrochemistry Communications, 2000, 2, 3944.

63. Yoshimoto, S.; Ono, Y.; Kuwahara, Y.; Nishiyama, K.; Taniguchi, I. Structural Changes of 4,4'-(Dithiodibutylene)dipyridine SAM on a $\mathrm{Au}(111)$ Electrode with Applied Potential and Solution pH and Influence of Alkyl Chain Length of Pyridine-Terminated Thiolate SAMs on Cytochrome $c$ Electrochemistry. J. Phys. Chem. $C$, 2016, 120, 15803-15813.

64. Yang, D. Y.; Morin, M. Chronoamperometric Study of the Reductive Desorption of Alkanethiol Self-assembled Monolayers, J. Electroanal. Chem., 1998, 441, 173-181.

65. Chi, Q.; Ford, M.J.; Halder, A.; Hush, N.S.; Reimers, J.R.; Marcuccio, S.; Ulstrup, J. Sulfur Ligand Mediated Electrochemistry of Gold Surfaces and Nanoparticles: What, How, and Why? Curr. Opin. Electrochemistry 2017,1, 715.

66. Reimers, J.R.; Ford, M.J.; Marcuccio, S.; Ulstrup, J.; Hush, N.S. Competition of van der Waals and Chemical Forces on Gold-Sulfur Surfaces and Nanoparticles. Nature Reviews Chemistry, 2017, 1, 0017.

67. Bell, R.P.; Davis, G.G. Kinetics of Bromination of some Enols and their Anions. J. Chem. Soc. 1965, 353-361.

68. Bell, R.P.; Dolman, D.G. Kinetics of Reactions of Olefins with Halogens in Aqueous Solition. 3. Reaction of Bromine with Acrylic Acid, Crotonic Acid, and Ethyl
Acrylate. J. Chem. Soc. B-Physical Organic 1968, 500-503.

69. Poirier, G. E. Butanethiol Self-assembly on $\mathrm{Au}(001)$ : The $1 \times 4 \mathrm{Au}$ Missing Row, $\mathrm{c}(2 \times 8)$ Molecular Lattice, J. Vac. Sci. Technol. B., 1996, 14, 1453-1460.

70. Yoshimoto, S. Stability and Structural Phase Transistions of Cobalt Porphyrin Adlayers on $\mathrm{Au}(100)$ Surfaces, Phys. Chem. Chem. Phys., 2013, 15, 12504-12509.

71. Yau, S.; Lee, Y.; Chang, C.; Dow, W. Revelation of the Spatial and Polymerization of Aniline on Au(100) Electrode by in situ Scanning Tunnelling Microscopy, Chem. Commun., 2009, 5737-5739.

72. Zhang, J.; Bilić, A.; Reimers, J. R.; Hush, N. S.; Ulstrup, J. Co-existence of Multiple Conformations in Cysteamine Monolayers on $\mathrm{Au}(111)$, J. Phys. Chem. B, 2005, 109, 1535515367.

73. Zhang, J.; Chi, Q.; Ulstrup, J.; Assembly Dynamics and Detailed Structure of 1Propanethiol Monolayers on $\mathrm{Au}(111)$ Surfaces Observed in Real-time by in situ STM, Langmuir, 2006, 22, 6203-6213.

74. Zhang, J.; Demetriou, A.; Welinder, A. C.; Albrecht, T.; Nichols, R. J.; Ulstrup, J. PotentialInduced Structural Transitions of Homocysteine Monolayers on $\mathrm{Au}(111)$ electrode Surface, Chem. Phys., 2005, 319, 210-221.

75. Zhang, L.; Kepp, K. P.; Ulstrup, J., Zhang, J. Redox Potentials and Electronic States of Iron Porphyrin IX Adsorbed on Single Crystal Gold Electrode Surfaces, Langmuir, 2018, 34, 36103618.

76. Yan, J.; Ouyang, R.; Jensen, P. S.; Ascic, E.; Tanner, D.; Mao, B.; Zhang, J.; Tang, C.; Hush, N. S.; Ulstrup, J. Reimers, J. R. 'Controlling the Stereochemistry and Regularity of Butanethiol Self-Assembled Monolayers on Au(111)', J. Am. Chem. Soc., 2014, 136, 17087-17094.

77. Wang, Y.; Chi, Q.; Zhang, J.; Hush, N. S.; Reimers J. R.; Ulstrup, J. 'Chain-Branching Control of the Atomic Structure of AlkanethiolBased Gold-Sulfur Interfaces', J. Am. Chem. Soc., 2011, 133, 14856-14859. 
78. Ouyang, R.; Yan, J.; Jensen, P.S.; Ascic, E.; Gan, S.; Tanner, D.; Mao, B.; Niu, L.; Zhang, J.; Tang, C.; Hush, N.S.; Reimers, J.R.; Ulstrup, J. Intermixed Atom and Surface Bound Adsorbates in Regular Selfassembled Monolayers of Racemic 2Butanethiol on $\mathrm{Au}(111)$. ChemPhysChem 2015, 16, 928-932.

79. Ignaczak, A.; Gomes, J.A.N.F. Theoretical Study of the Interaction of Water Molecules with the $\mathrm{Cu}(100), \operatorname{Ag}(100)$ and $\mathrm{Au}(100)$ surfaces. $J$. Electroanal. Chem., 1997, 420, 209-218.

80. Popa, T., Ting, E. C. M.; Paci, I. Chiral Effects in Amino Acid Adsorption on $\mathrm{Au}(111)$ : A Comparison of Cysteine, Homocysteine and Methionine. Surface Science, 2014, 629, 20-27.

81. G.A. Attard, G.A.; Baines, C. 1998. Surfaces, Oxford Chemistry Primers, Oxford University Press, Oxford.

82. S.J. Jenkins, 2018. Chirality at Solid Surfaces, Wiley 2018

83. Lyuksyutov, I.F.; Naumovets, A.G.;

Pokrovsky, V. Two-dimensional Crystals.

1992, Academic Press, San Diego and London. 


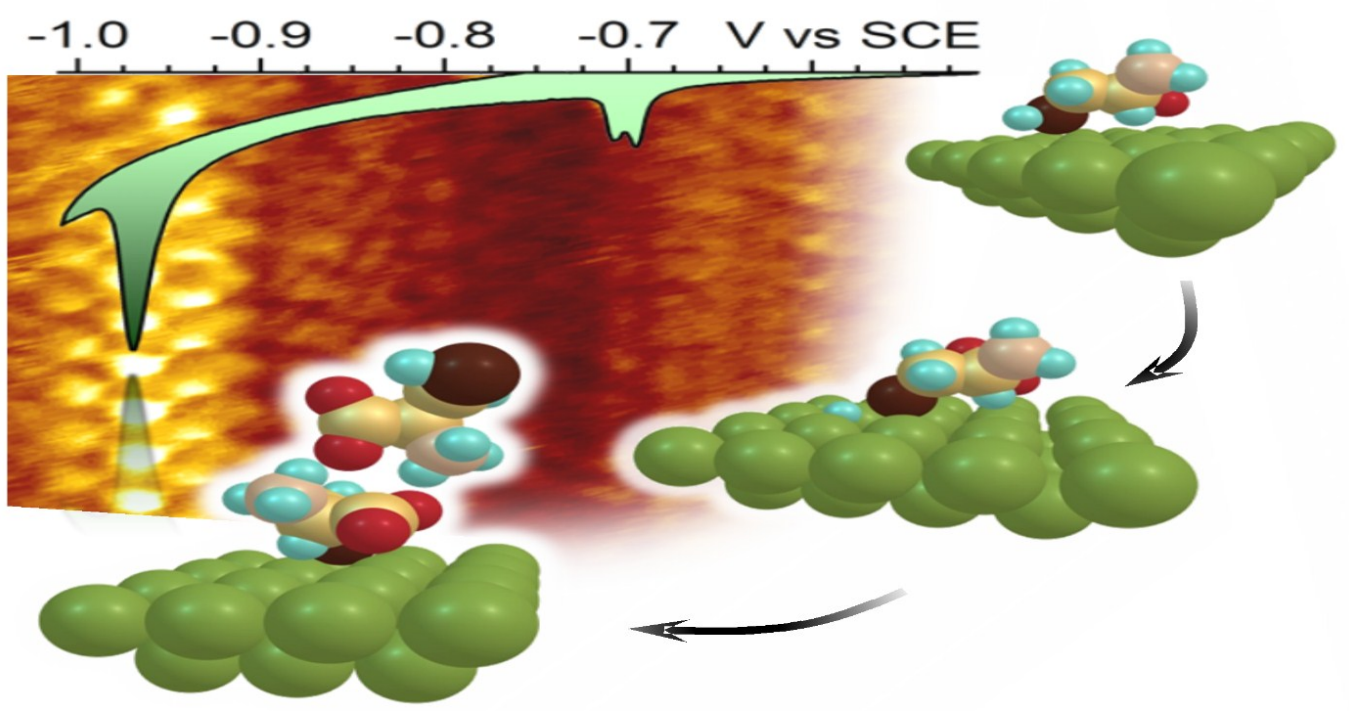

